\title{
Partial Sums of Two Quartic q-Series
}

Wenchang $\mathrm{CHU}^{\dagger}$ and Chenying $W A N G \ddagger$

$\dagger$ Dipartimento di Matematica, Università degli Studi di Salento, Lecce-Arnesano P. O. Box 193, Lecce 73100, Italy

E-mail: chu.wenchang@unile.it

$\ddagger$ College of Mathematics and Physics, Nanjing University of Information Science and Technology, Nanjing 210044, P. R. China

E-mail: wang.chenying@163.com

Received January 20, 2009, in final form April 17, 2009; Published online April 22, 2009 doi:10.3842/SIGMA.2009.050

Abstract. The partial sums of two quartic basic hypergeometric series are investigated by means of the modified Abel lemma on summation by parts. Several summation and transformation formulae are consequently established.

Key words: basic hypergeometric series ( $q$-series); well-poised $q$-series; quadratic $q$-series; cubic $q$-series; quartic $q$-series; the modified Abel lemma on summation by parts

2000 Mathematics Subject Classification: 33D15; 05A15

\section{Introduction and motivation}

For two indeterminate $x$ and $q$, the shifted-factorial of $x$ with base $q$ is defined by

$$
(x ; q)_{0}=1 \quad \text { and } \quad(x ; q)_{n}=(1-x)(1-x q) \cdots\left(1-x q^{n-1}\right) \quad \text { for } \quad n \in \mathbb{N} .
$$

When $|q|<1$, we have two well-defined infinite products

$$
(x ; q)_{\infty}=\prod_{k=0}^{\infty}\left(1-q^{k} x\right) \quad \text { and } \quad(x ; q)_{n}=(x ; q)_{\infty} /\left(x q^{n} ; q\right)_{\infty} .
$$

The product and fraction of shifted factorials are abbreviated respectively to

$$
\begin{aligned}
& {[\alpha, \beta, \ldots, \gamma ; q]_{n}=(\alpha ; q)_{n}(\beta ; q)_{n} \cdots(\gamma ; q)_{n},} \\
& {\left[\begin{array}{c}
\alpha, \beta, \ldots, \gamma \\
A, B, \ldots, C
\end{array} \mid q\right]_{n}=\frac{(\alpha ; q)_{n}(\beta ; q)_{n} \cdots(\gamma ; q)_{n}}{(A ; q)_{n}(B ; q)_{n} \cdots(C ; q)_{n}} .}
\end{aligned}
$$

Following Gasper-Rahman [12], the basic hypergeometric series is defined by

$$
{ }_{1+r} \phi_{s}\left[\begin{array}{c}
a_{0}, a_{1}, \ldots, a_{r} \\
b_{1}, \ldots, b_{s}
\end{array} \mid q ; z\right]=\sum_{n=0}^{\infty}\left\{(-1)^{n} q^{\left(\begin{array}{c}
n \\
2
\end{array}\right)}\right\}^{s-r}\left[\begin{array}{c}
a_{0}, a_{1}, \ldots, a_{r} \\
q, b_{1}, \ldots, b_{s}
\end{array} \mid q\right]_{n} z^{n},
$$

where the base $q$ will be restricted to $|q|<1$ for nonterminating $q$-series. For its connections to special functions and orthogonal polynomials, the reader can refer, for example, to the monograph written by Andrews-Askey-Roy [2] and the paper by Koornwinder [15].

In the theory of basic hypergeometric series, there are several important classes, for example, well-poised [3], quadratic [13, 17], cubic [16] and quartic [10,11] series. To our knowledge, there are four quartic series which can be displayed as follows:

$$
F_{n}(a, b, d):=\sum_{k=0}^{n-1}\left(1-q^{5 k} a\right)\left[\begin{array}{c}
b, d \\
q^{3} a / b^{2} d^{2}
\end{array} \mid q\right]_{k} \frac{[q a / b d ; q]_{3 k}}{\left[b d, b d / q, q b d ; q^{2}\right]_{k}}\left[\begin{array}{c}
b^{2} d^{2} / q^{2} \\
q^{4} a / b, q^{4} a / d
\end{array} \mid q^{4}\right]_{k} q^{k}
$$




$$
\begin{aligned}
G_{n}(a, c, e) & :=\sum_{k=0}^{n-1}\left(1-q^{5 k} a\right)\left[\begin{array}{c}
c^{2} e^{2} / q^{2} a^{3} \\
q a / c, q a / e
\end{array} \mid q\right]_{k} \frac{\left[q a^{2} / c e, q^{2} a^{2} / c e, q^{3} a^{2} / c e ; q^{2}\right]_{k}\left[\begin{array}{c}
c, e \\
(c e / a ; q)_{3 k}
\end{array} q^{6} a^{4} / c^{2} e^{2} \mid q^{4}\right]_{k} q^{k}}{U_{n}(a, b, d)}:=\sum_{k=0}^{n-1}\left(1-q^{5 k} a\right) \frac{\left(q^{2} a / b d ; q\right)_{k}}{\left(b d ; q^{2}\right)_{2 k}}\left[\begin{array}{c}
b, d \\
q^{5} a^{2} / b^{2} d^{2} \mid q^{2}
\end{array}\right]_{k} \frac{\left(q^{3} a^{2} / b d ; q^{6}\right)_{k}}{\left(-b d / q^{3} a\right)^{k}}\left[\begin{array}{c}
b^{2} d^{2} / q^{3} a \\
q^{3} a / b, q^{3} a / d
\end{array} \mid q^{3}\right]_{k} q^{\left(\begin{array}{c}
k \\
2
\end{array}\right)} ; \\
V_{n}(a, c, e) & :=\sum_{k=0}^{n-1}\left(1-q^{5 k} a\right) \frac{\left(a^{2} / c e ; q^{2}\right)_{2 k}}{(q c e / a ; q)_{k}}\left[\begin{array}{c}
q c^{2} e^{2} / a^{2} \\
q a / c, q a / e
\end{array} \mid q^{2}\right]_{k} \frac{(-a / c e)^{k}}{\left(q^{5} c e ; q^{6}\right)_{k}}\left[\begin{array}{c}
q c, q e \\
q^{2} a^{3} / c^{2} e^{2}
\end{array} \mid q^{3}\right]_{k} q^{-\left(\begin{array}{c}
k \\
2
\end{array}\right)}
\end{aligned}
$$

By means of the series rearrangement, Gasper and Rahman [10, 11, 12] discovered several summation and transformation formulae for the nonterminating special cases of $F_{n}(a, b, d)$ and $U_{n}(a, b, d)$. The terminating series identities for the last four sums have been established by Chu [4] and Chu-Wang [8], respectively, through inversion techniques and Abel's lemma on summation by parts. For the partial sums $F_{n}(a, b, d)$ and $G_{n}(a, c, e)$, the present authors [7] recently derived several useful reciprocal relations and transformation formulae in terms of wellpoised series.

The purpose of this paper is to investigate the remaining two partial sums $U_{n}(a, b, d)$ and $V_{n}(a, c, e)$. By utilizing the modified Abel lemma on summation by parts, we shall show six unusual transformation formulae with two between them and other four expressing $U_{n}(a, b, d)$ and $V_{n}(a, c, e)$ as partial sums of quadratic and cubic series. Several new and known terminating as well as nonterminating series identities are consequently obtained as particular instances.

In order to make the paper self-contained, we reproduce Abel's lemma on summation by parts (cf. $[5,6,7,8]$ ) as follows. For an arbitrary complex sequence $\left\{\tau_{k}\right\}$, define the backward and forward difference operators $\nabla$ and $\Delta$, respectively, by

$$
\nabla \tau_{k}=\tau_{k}-\tau_{k-1} \quad \text { and } \quad \Delta \tau_{k}=\tau_{k+1}-\tau_{k}
$$

Then Abel's lemma on summation by parts can be modified as follows:

$$
\sum_{k=0}^{n-1} B_{k} \nabla A_{k}=\left\{A_{n-1} B_{n}-A_{-1} B_{0}\right\}-\sum_{k=0}^{n-1} A_{k} \Delta B_{k} .
$$

This can be considered as the discrete counterpart for the integral formula

$$
\int_{a}^{b} f(x) g^{\prime}(x) \mathrm{d} x=\{f(b) g(b)-f(a) g(a)\}-\int_{a}^{b} f^{\prime}(x) g(x) \mathrm{d} x .
$$

In fact, it is almost trivial to check the following expression

$$
\sum_{k=0}^{n-1} B_{k} \nabla A_{k}=\sum_{k=0}^{n-1} B_{k}\left\{A_{k}-A_{k-1}\right\}=\sum_{k=0}^{n-1} A_{k} B_{k}-\sum_{k=0}^{n-1} A_{k-1} B_{k}
$$

Replacing $k$ by $k+1$ for the last sum, we can reformulate the equation as follows

$$
\begin{aligned}
\sum_{k=0}^{n-1} B_{k} \nabla A_{k} & =A_{n-1} B_{n}-A_{-1} B_{0}+\sum_{k=0}^{n-1} A_{k}\left\{B_{k}-B_{k+1}\right\} \\
& =A_{n-1} B_{n}-A_{-1} B_{0}-\sum_{k=0}^{n-1} A_{k} \Delta B_{k}
\end{aligned}
$$

which is exactly the equality stated in the modified Abel lemma. 
Throughout the paper, if $W_{n}$ is used to denote the partial sum of some $q$-series, then the corresponding letter $W$ without subscript will stand for the limit of $W_{n}$ (if it exists of course) when $n \rightarrow \infty$.

When applying the modified Abel lemma on summation by parts to deal with hypergeometric series, the crucial step lies in finding shifted factorial fractions $\left\{A_{k}, B_{k}\right\}$ so that their differences are expressible as ratios of linear factors. This has not been an easy task, even though it is indeed routine to factorize $\left\{A_{k}, B_{k}\right\}$ once they are figured out. Specifically for $U_{n}(a, b, d)$ and $V_{n}(a, b, d)$, we shall devise three well-poised difference pairs for each partial sum. This is based on numerous attempts to detect $A_{k}$ and $B_{k}$ sequences such that not only their differences turn out to be factorizable, but also their combinations match exactly the summands displayed in $U_{n}(a, b, d)$ and $V_{n}(a, b, d)$.

The contents of the paper will be organized as follows. In the second section, $U_{n}(a, b, d)$ will be reformulated through the modified Abel lemma on summation by parts, which lead to three transformations of $U_{n}(a, b, d)$ into partial sums of quadratic, cubic and quartic series. Then the third section will be devoted to the transformation formulae of $V_{n}(a, b, d)$ in terms of partial sums of quadratic, cubic and quartic series. These transformations on $U_{n}(a, b, d)$ and $V_{n}(a, b, d)$ will recover several known identities appeared in Chu-Wang [4, 8] and Gasper-Rahman [12], and yield a few additional new summation formulae.

\section{Transformation and summation formulae for $U_{n}(a, b, d)$}

In this section, we shall investigate the partial sum of quartic $q$-series $U_{n}(a, b, d)$. Three transformation formulae will be established from $U_{n}(a, b, d)$ to quadratic, cubic and another quartic series, unlike those for $F_{n}(a, b, d)$ and $G_{n}(a, c, e)$ shown in [7], where reciprocal relations and transformations into well-poised partial sums have been derived. As particular cases of $U_{n}(a, b, d)$, four nonterminating series will be evaluated, including two that appeared in GasperRahman's book [12].

In order for the reader to gain an immediate insight into the well-poised structure, we reformulate the series $U_{n}(a, b, d)$ in the following manner:

$$
\begin{aligned}
U_{n}(a, b, d)= & \sum_{k=0}^{n-1}\left(1-q^{5 k} a\right) \frac{\left[b, d ; q^{2}\right]_{k}}{\left[q^{3} a / b, q^{3} a / d ; q^{3}\right]_{k}} \frac{\left(b^{2} d^{2} / q^{3} a ; q^{3}\right)_{k}}{\left(q^{5} a^{2} / b^{2} d^{2} ; q^{2}\right)_{k}} q^{k} \\
& \times \frac{\left[q^{2} a / b d, q^{2} a / b d ; q\right]_{k}}{\left[b d, q^{2} b d ; q^{4}\right]_{k}} \frac{\left(q^{3} a^{2} / b d ; q^{6}\right)_{k}}{\left(b d / q^{2} a ; q^{-1}\right)_{k}} .
\end{aligned}
$$

\subsection{Quartic series to quadratic series}

Let $\mathrm{A}_{k}$ and $\mathrm{B}_{k}$ be defined by

$$
\begin{aligned}
\mathrm{A}_{k} & =\frac{\left[q^{3} a / b d, q^{5} a / b d ; q\right]_{k}\left(b^{3} d^{3} / q^{7} a^{2} ; q^{2}\right)_{k}\left(q^{9} a^{2} / b d ; q^{6}\right)_{k}}{\left[b d, q^{2} b d ; q^{4}\right]_{k}\left(q^{12} a^{3} / b^{3} d^{3} ; q^{3}\right)_{k}\left(b d / q^{4} a ; q^{-1}\right)_{k}}, \\
\mathrm{~B}_{k} & =\frac{\left[b, d ; q^{2}\right]_{k}}{\left[q^{3} a / b, q^{3} a / d ; q^{3}\right]_{k}} \frac{\left[b^{2} d^{2} / q^{6} a, q^{12} a^{3} / b^{3} d^{3} ; q^{3}\right]_{k}}{\left[q^{9} a^{2} / b^{2} d^{2}, b^{3} d^{3} / q^{9} a^{2} ; q^{2}\right]_{k}} .
\end{aligned}
$$

We can easily show the relations

$$
\begin{aligned}
& \varpi:=\mathrm{A}_{-1} \mathrm{~B}_{0}=\frac{a\left(1-q^{2} / b d\right)\left(1-q^{4} / b d\right)\left(1-q^{3} a / b d\right)\left(1-q^{9} a^{3} / b^{3} d^{3}\right)}{\left(1-q^{2} a / b d\right)\left(1-q^{4} a / b d\right)\left(1-q^{3} a^{2} / b d\right)\left(1-q^{9} a^{2} / b^{3} d^{3}\right)}, \\
& \mathcal{R}:=\frac{\mathrm{A}_{n-1} \mathrm{~B}_{n}}{\mathrm{~A}_{-1} \mathrm{~B}_{0}}=\frac{1-q^{9+3 n} a^{3} / b^{3} d^{3}}{1-q^{9} a^{3} / b^{3} d^{3}}\left[\begin{array}{c}
b, d \\
q^{9} a^{2} / b^{2} d^{2}
\end{array} \mid q^{2}\right]_{n}
\end{aligned}
$$




$$
\times \frac{\left[q^{2} a / b d, q^{4} a / b d ; q\right]_{n}}{\left(b d / q^{4} ; q^{2}\right)_{2 n}}\left[\begin{array}{c}
b^{2} d^{2} / q^{6} a \\
q^{3} a / b, q^{3} a / d
\end{array} \mid q^{3}\right]_{n} \frac{\left(q^{3} a^{2} / b d ; q^{6}\right)_{n}}{\left(b d / q^{3} a ; q^{-1}\right)_{n}}
$$

and calculate the finite differences

$$
\begin{aligned}
\nabla \mathrm{A}_{k}= & \frac{\left(1-q^{5 k} a\right)\left(1-q^{6-3 k} a / b^{2} d^{2}\right)\left(1-q^{2 k+5} a^{2} / b^{2} d^{2}\right)\left(1-q^{2 k+7} a^{2} / b^{2} d^{2}\right)}{\left(1-q^{2} a / b d\right)\left(1-q^{4} a / b d\right)\left(1-q^{3} a^{2} / b d\right)\left(1-q^{9} a^{2} / b^{3} d^{3}\right)} \\
& \times \frac{\left[q^{2} a / b d, q^{4} a / b d ; q\right]_{k}\left(b^{3} d^{3} / q^{9} a^{2} ; q^{2}\right)_{k}\left(q^{3} a^{2} / b d ; q^{6}\right)_{k}}{\left[b d, q^{2} b d ; q^{4}\right]_{k}\left(q^{12} a^{3} / b^{3} d^{3} ; q^{3}\right)_{k}\left(b d / q^{4} a ; q^{-1}\right)_{k}}, \\
\Delta \mathrm{B}_{k}= & -\frac{\left(1-q^{3+5 k} a\right)\left(1-q^{3+k} a / b d\right)\left(1-q^{9} a^{2} / b^{2} d^{3}\right)\left(1-q^{9} a^{2} / b^{3} d^{2}\right)}{\left(1-q^{3} a / b\right)\left(1-q^{3} a / d\right)\left(1-q^{9} a^{2} / b^{2} d^{2}\right)\left(1-q^{9} a^{2} / b^{3} d^{3}\right)} \\
& \times\left[\begin{array}{c}
b, d \\
\left.q^{11} a^{2} / b^{2} d^{2}, b^{3} d^{3} / q^{7} a^{2} \mid q^{2}\right]_{k}\left[\begin{array}{c}
b^{2} d^{2} / q^{6} a, q^{12} a^{3} / b^{3} d^{3} \\
q^{6} a / b,
\end{array} q^{6} a / d\right.
\end{array}\right]_{k} q^{2 k} .
\end{aligned}
$$

According to the modified Abel lemma on summation by parts, the finite $U$-sum can be reformulated as follows:

$$
\begin{aligned}
U_{n}(a, b, d) & \frac{\left(1-q^{5} a^{2} / b^{2} d^{2}\right)\left(1-q^{7} a^{2} / b^{2} d^{2}\right)\left(1-q^{6} a / b^{2} d^{2}\right)}{\left(1-q^{2} a / b d\right)\left(1-q^{4} a / b d\right)\left(1-q^{3} a^{2} / b d\right)\left(1-q^{9} a^{2} / b^{3} d^{3}\right)} \\
= & \sum_{k=0}^{n-1} \mathrm{~B}_{k} \nabla \mathrm{A}_{k}=\varpi\{\mathcal{R}-1\}-\sum_{k=0}^{n-1} \mathrm{~A}_{k} \Delta \mathrm{B}_{k} .
\end{aligned}
$$

Observing that there holds the equality for the last partial sum

$$
-\sum_{k=0}^{n-1} \mathrm{~A}_{k} \Delta \mathrm{B}_{k}=\frac{U_{n}\left(q^{3} a, b, d\right)\left(1-q^{3} a / b d\right)\left(1-q^{9} a^{2} / b^{2} d^{3}\right)\left(1-q^{9} a^{2} / b^{3} d^{2}\right)}{\left(1-q^{3} a / b\right)\left(1-q^{3} a / d\right)\left(1-q^{9} a^{2} / b^{2} d^{2}\right)\left(1-q^{9} a^{2} / b^{3} d^{3}\right)},
$$

we derive after some simplification the recurrence relation

$$
\begin{aligned}
U_{n}(a, b, d)= & U_{n}\left(q^{3} a, b, d\right) \frac{\left(q^{2} a / b d ; q\right)_{3}\left(1-q^{3} a^{2} / b d\right)\left(1-q^{9} a^{2} / b^{2} d^{3}\right)\left(1-q^{9} a^{2} / b^{3} d^{2}\right)}{\left(q^{5} a^{2} / b^{2} d^{2} ; q^{2}\right)_{3}\left(1-q^{3} a / b\right)\left(1-q^{3} a / d\right)\left(1-q^{6} a / b^{2} d^{2}\right)} \\
& -a\{1-\mathcal{R}(a, b, d)\} \frac{\left(1-q^{2} / b d\right)\left(1-q^{4} / b d\right)\left(1-q^{3} a / b d\right)\left(1-q^{9} a^{3} / b^{3} d^{3}\right)}{\left(1-q^{5} a^{2} / b^{2} d^{2}\right)\left(1-q^{7} a^{2} / b^{2} d^{2}\right)\left(1-q^{6} a / b^{2} d^{2}\right)} .
\end{aligned}
$$

Iterating this equation $m$-times, we get the following expression

$$
\begin{aligned}
& U_{n}(a, b, d)=U_{n}\left(q^{3 m} a, b, d\right) \frac{\left(q^{2} a / b d ; q\right)_{3 m}\left[q^{3} a^{2} / b d, q^{9} a^{2} / b^{2} d^{3}, q^{9} a^{2} / b^{3} d^{2} ; q^{6}\right]_{m}}{\left(q^{5} a^{2} / b^{2} d^{2} ; q^{2}\right)_{3 m}\left[q^{3} a / b, q^{3} a / d, q^{6} a / b^{2} d^{2} ; q^{3}\right]_{m}} \\
& -\frac{a\left(1-q^{2} / b d\right)\left(1-q^{4} / b d\right)\left(1-q^{3} a / b d\right)}{\left(1-q^{5} a^{2} / b^{2} d^{2}\right)\left(1-q^{7} a^{2} / b^{2} d^{2}\right)\left(1-q^{6} a / b^{2} d^{2}\right)} \\
& \times \sum_{k=0}^{m-1}\left(1-q^{9+9 k} a^{3} / b^{3} d^{3}\right)\left[\left.\begin{array}{l}
q^{2} a / b d, q^{4} a / b d, q^{6} a / b d \\
q^{3} a / b, q^{3} a / d, q^{9} a / b^{2} d^{2}
\end{array}\right|^{3}\right]_{k} q^{3 k} \\
& \times\left\{1-\mathcal{R}\left(q^{3 k} a, b, d\right)\right\}\left[\begin{array}{c}
q^{3} a^{2} / b d, q^{9} a^{2} / b^{2} d^{3}, q^{9} a^{2} / b^{3} d^{2} \\
q^{9} a^{2} / b^{2} d^{2}, q^{11} a^{2} / b^{2} d^{2}, q^{13} a^{2} / b^{2} d^{2} \mid q^{6}
\end{array}\right]_{k} .
\end{aligned}
$$

Writing explicitly the $\mathcal{R}$-function by separating $k$ and $n$ factorials

$$
\begin{aligned}
\mathcal{R}\left(q^{3 k} a, b, d\right)= & \frac{1-q^{9+3 n+9 k} a^{3} / b^{3} d^{3}}{1-q^{9+9 k} a^{3} / b^{3} d^{3}}\left[\begin{array}{c}
b, d \\
q^{9+6 k} a^{2} / b^{2} d^{2}
\end{array} \mid q^{2}\right]_{n} \\
& \times \frac{\left[q^{2+3 k} a / b d, q^{4+3 k} a / b d ; q\right]_{n}}{\left(b d / q^{4} ; q^{2}\right)_{2 n}}\left[\begin{array}{c}
q^{-6-3 k} b^{2} d^{2} / a \\
q^{3+3 k} a / b, q^{3+3 k} a / d
\end{array} \mid q^{3}\right]_{n} \frac{\left(q^{3+6 k} a^{2} / b d ; q^{6}\right)_{n}}{\left(q^{-3-3 k} b d / a ; q^{-1}\right)_{n}}
\end{aligned}
$$




$$
\begin{aligned}
= & \frac{\left[q^{2} a / b d, q^{4} a / b d ; q\right]_{n}}{\left(b d / q^{4} ; q^{2}\right)_{2 n}}\left[\begin{array}{c}
b, d \\
q^{9} a^{2} / b^{2} d^{2} \mid q^{2}
\end{array}\right]_{n}\left[\begin{array}{c}
b^{2} d^{2} / q^{6} a \\
q^{3} a / b, q^{3} a / d
\end{array} \mid q^{3}\right]_{n} \frac{\left(q^{3} a^{2} / b d ; q^{6}\right)_{n}}{\left(b d / q^{3} a ; q^{-1}\right)_{n}} \\
& \times \frac{1-q^{9+3 n+9 k} a^{3} / b^{3} d^{3}}{1-q^{9+9 k} a^{3} / b^{3} d^{3}}\left[\begin{array}{c}
q^{3} a / b, q^{3} a / d, q^{9} a / b^{2} d^{2} \\
q^{2} a / b d, q^{4} a / b d, q^{6} a / b d
\end{array} \mid q^{3}\right]_{k} \frac{\left(q^{3+6 n} a^{2} / b d ; q^{6}\right)_{k}}{\left(q^{3} a^{2} / b d ; q^{6}\right)_{k}} \\
& \times \frac{\left(q^{9} a^{2} / b^{2} d^{2} ; q^{2}\right)_{3 k}}{\left(q^{9+2 n} a^{2} / b^{2} d^{2} ; q^{2}\right)_{3 k}}\left[\begin{array}{c}
q^{2+n} a / b d, q^{4+n} a / b d, q^{6+n} a / b d \\
q^{3+3 n} a / b, q^{3+3 n} a / d, q^{9-3 n} a / b^{2} d^{2}
\end{array} \mid q^{3}\right]_{k},
\end{aligned}
$$

and then defining the partial sum of quadratic series in base $q^{3}$ by

$$
\begin{aligned}
& U_{m}^{\diamond}(a, b, d)=\sum_{k=0}^{m-1}\left(1-q^{9+9 k} a^{3} / b^{3} d^{3}\right)\left[\begin{array}{l}
q^{2} a / b d, q^{4} a / b d, q^{6} a / b d \\
q^{3} a / b, q^{3} a / d, q^{9} a / b^{2} d^{2}
\end{array} \mid q^{3}\right]_{k} \\
& \times\left[\begin{array}{c}
q^{3} a^{2} / b d, q^{9} a^{2} / b^{2} d^{3}, q^{9} a^{2} / b^{3} d^{2} \\
q^{9} a^{2} / b^{2} d^{2}, q^{11} a^{2} / b^{2} d^{2}, q^{13} a^{2} / b^{2} d^{2}
\end{array} \mid q^{6}\right]_{k} q^{3 k},
\end{aligned}
$$

we derive the following transformation formula.

Theorem 1 (Transformation between quartic and quadratic series).

$$
\begin{aligned}
U_{n}(a, b, d)- & U_{n}\left(q^{3 m} a, b, d\right) \frac{\left(q^{2} a / b d ; q\right)_{3 m}\left[q^{3} a^{2} / b d, q^{9} a^{2} / b^{2} d^{3}, q^{9} a^{2} / b^{3} d^{2} ; q^{6}\right]_{m}}{\left(q^{5} a^{2} / b^{2} d^{2} ; q^{2}\right)_{3 m}\left[q^{3} a / b, q^{3} a / d, q^{6} a / b^{2} d^{2} ; q^{3}\right]_{m}} \\
= & \frac{\left(1-q^{3} a / b d\right)\left(1-b d / q^{2}\right)\left(1-b d / q^{4}\right)}{\left(1-q^{5} a^{2} / b^{2} d^{2}\right)\left(1-q^{7} a^{2} / b^{2} d^{2}\right)\left(1-b^{2} d^{2} / q^{6} a\right)} \\
& \times\left\{U_{m}^{\diamond}(a, b, d)-U_{m}^{\diamond}\left(q^{5 n} a, q^{2 n} b, q^{2 n} d\right) \frac{\left(q^{3} a^{2} / b d ; q^{6}\right)_{n}}{\left(b d / q^{3} a ; q^{-1}\right)_{n}}\right. \\
& \left.\times\left[\begin{array}{c}
b, d \\
q^{9} a^{2} / b^{2} d^{2}
\end{array} \mid q^{2}\right]_{n} \frac{\left[q^{2} a / b d, q^{4} a / b d ; q\right]_{n}}{\left(b d / q^{4} ; q^{2}\right)_{2 n}}\left[\begin{array}{c}
b^{2} d^{2} / q^{6} a \\
q^{3} a / b, q^{3} a / d
\end{array} \mid q^{3}\right]_{n}\right\} .
\end{aligned}
$$

By means of the Weierstrass $M$-test on uniformly convergent series (cf. Stromberg [19, p. 141]), we can compute the following limit

$$
\lim _{m, n \rightarrow \infty} U_{n}\left(q^{3 m} a, b, d\right)=\sum_{k=0}^{\infty}(b d)^{k} \frac{\left[b, d ; q^{2}\right]_{k}}{\left(b d ; q^{2}\right)_{2 k}} q^{4\left(\begin{array}{c}
k \\
2
\end{array}\right) .}
$$

Letting $m, n \rightarrow \infty$ in Theorem 1 , we obtain the transformation formula.

\section{Proposition 2 (Nonterminating series transformation).}

$$
\begin{aligned}
U(a, b, d)= & \frac{\left(1-q^{3} a / b d\right)\left(1-b d / q^{2}\right)\left(1-b d / q^{4}\right)}{\left(1-q^{5} a^{2} / b^{2} d^{2}\right)\left(1-q^{7} a^{2} / b^{2} d^{2}\right)\left(1-b^{2} d^{2} / q^{6} a\right)} U^{\diamond}(a, b, d) \\
& +\frac{\left(q^{2} a / b d ; q\right)_{\infty}\left[q^{3} a^{2} / b d, q^{9} a^{2} / b^{2} d^{3}, q^{9} a^{2} / b^{3} d^{2} ; q^{6}\right]_{\infty}}{\left(q^{5} a^{2} / b^{2} d^{2} ; q^{2}\right)_{\infty}\left[q^{3} a / b, q^{3} a / d, q^{6} a / b^{2} d^{2} ; q^{3}\right]_{\infty}} \sum_{k=0}^{\infty}(b d)^{k} \frac{\left[b, d ; q^{2}\right]_{k}}{\left(b d ; q^{2}\right)_{2 k}} q^{4}\left(\begin{array}{c}
k \\
2
\end{array}\right) .
\end{aligned}
$$

When $b d=q^{2+2 \delta}$ with $\delta=0,1$, this proposition results in

$$
\begin{aligned}
U\left(a, b, q^{2+2 \delta} / b\right)= & {\left[\begin{array}{c}
a, q^{1-2 \delta} a \\
q^{3} a / b, q^{1-2 \delta} a b
\end{array} \mid q^{3}\right]_{\infty}\left[\begin{array}{c}
q^{3-6 \delta} a^{2} b, q^{5-4 \delta} a^{2} / b \\
q^{3-6 \delta} a^{2}, q^{5-4 \delta} a^{2}
\end{array} \mid q^{6}\right]_{\infty} } \\
& \times \sum_{k=0}^{\infty} \frac{\left[b, q^{2+2 \delta} / b ; q^{2}\right]_{k}}{\left(q^{2+2 \delta} ; q^{2}\right)_{2 k}} q^{4\left(\begin{array}{c}
k \\
2
\end{array}\right)+(2+2 \delta) k} .
\end{aligned}
$$


Taking $a=q^{1+4 \delta}$ in this equation and noting that the initial condition

$$
U\left(q^{1+4 \delta}, b, q^{2+2 \delta} / b\right)=1-q^{1+4 \delta},
$$

we recover the following formula due to Andrews [1, equation (4.6)] and Ismail-Stanton [14, Proposition 6]

$$
\sum_{k=0}^{\infty} \frac{\left[b, q^{2+2 \delta} / b ; q^{2}\right]_{k}}{\left(q^{2+2 \delta} ; q^{2}\right)_{2 k}} q^{4\left(\begin{array}{c}
k \\
2
\end{array}\right)+(2+2 \delta) k}=\left[\begin{array}{c}
q^{2+2 \delta} b, q^{4+4 \delta} / b \\
q^{2+2 \delta}, q^{4+4 \delta}
\end{array} \mid q^{6}\right]_{\infty},
$$

which leads, under the replacement $a \rightarrow q^{2 \delta} a$, to the nonterminating series formula.

Corollary 3 (Gasper-Rahman [12, Exercise 3.29(ii),(iii)]).

$$
\begin{gathered}
\sum_{k=0}^{\infty} \frac{1-q^{5 k+2 \delta} a}{1-q^{2 \delta} a}\left[\begin{array}{c}
b, q^{2+2 \delta} / b \\
q a^{2}
\end{array} \mid q^{2}\right]_{k} \frac{(a ; q)_{k}\left(q^{1+2 \delta} / a ; q^{3}\right)_{k}\left(q^{1+2 \delta} a^{2} ; q^{6}\right)_{k}}{\left(q^{2+2 \delta} ; q^{2}\right)_{2 k}\left[q a b, q^{3+2 \delta} a / b ; q^{3}\right]_{k}} q^{\left(\begin{array}{c}
k+1 \\
2
\end{array}\right)(-a)^{k}} \\
=\left[\begin{array}{c}
q a, q^{3+2 \delta} a \\
q a b, q^{3+2 \delta} a / b
\end{array} \mid q^{3}\right]_{\infty}\left[\begin{array}{c}
q^{5} a^{2} / b, q^{3-2 \delta} a^{2} b, q^{2+2 \delta} b, q^{4+4 \delta} / b \\
q^{5} a^{2}, q^{3-2 \delta} a^{2}, q^{2+2 \delta}, q^{4+4 \delta}
\end{array} q^{6}\right]_{\infty} .
\end{gathered}
$$

\subsection{Quartic series to cubic series}

Define two sequences by

$$
\begin{aligned}
\mathrm{A}_{k} & =\frac{\left[q^{3} a / b d, b d^{2} / q^{4} a ; q\right]_{k}\left(q^{2} b ; q^{2}\right)_{k}\left(q^{9} a^{2} / b d ; q^{6}\right)_{k}}{\left[q^{2} b d, q^{9} a^{2} / b d^{2} ; q^{4}\right]_{k}\left(q^{3} a / b ; q^{3}\right)_{k}\left(b d / q^{4} a ; q^{-1}\right)_{k}}, \\
\mathrm{~B}_{k} & =\frac{\left(q^{4} a / b d ; q\right)_{k}\left(d / q^{2} ; q^{2}\right)_{k}\left(b^{2} d^{2} / q^{3} a ; q^{3}\right)_{k}\left(q^{9} a^{2} / b d^{2} ; q^{4}\right)_{k}}{\left(b d ; q^{4}\right)_{k}\left(q^{6} a / d ; q^{3}\right)_{k}\left(q^{7} a^{2} / b^{2} d^{2} ; q^{2}\right)_{k}\left(b d^{2} / q^{5} a ; q\right)_{k}} .
\end{aligned}
$$

It is not hard to check the relations

$$
\begin{aligned}
\varpi:= & \mathrm{A}_{-1} \mathrm{~B}_{0}=\frac{\left(1-b d / q^{2}\right)\left(1-q^{5} a^{2} / b d^{2}\right)(1-a / b)\left(1-b d / q^{3} a\right)}{\left(1-q^{2} a / b d\right)\left(1-b d^{2} / q^{5} a\right)(1-b)\left(1-q^{3} a^{2} / b d\right)} \\
\mathcal{R}:= & \frac{\mathrm{A}_{n-1} \mathrm{~B}_{n}}{\mathrm{~A}_{-1} \mathrm{~B}_{0}}=\frac{1-q^{5+4 n} a^{2} / b d^{2}}{1-q^{5} a^{2} / b d^{2}}\left[\begin{array}{c}
b, d / q^{2} \\
q^{7} a^{2} / b^{2} d^{2}
\end{array} \mid q^{2}\right]_{n} \\
& \times \frac{\left[q^{2} a / b d, q^{4} a / b d ; q\right]_{n}}{\left(b d / q^{2} ; q^{2}\right)_{2 n}}\left[\begin{array}{c}
b^{2} d^{2} / q^{3} a \\
a / b, q^{6} a / d
\end{array} \mid q^{3}\right]_{n} \frac{\left(q^{3} a^{2} / b d ; q^{6}\right)_{n}}{\left(b d / q^{3} a ; q^{-1}\right)_{n}}
\end{aligned}
$$

and compute the finite differences

$$
\begin{aligned}
& \nabla \mathrm{A}_{k}=\frac{\left(1-q^{5 k} a\right)\left(1-q^{2-2 k} / d\right)\left(1-q^{5+2 k} a^{2} / b^{2} d^{2}\right)\left(1-q^{3+3 k} a / d\right)}{\left(1-q^{2} a / b d\right)\left(1-q^{5} a / b d^{2}\right)(1-b)\left(1-q^{3} a^{2} / b d\right)} \\
& \times \frac{\left[q^{2} a / b d, b d^{2} / q^{5} a ; q\right]_{k}\left(b ; q^{2}\right)_{k}\left(q^{3} a^{2} / b d ; q^{6}\right)_{k}}{\left[q^{2} b d, q^{9} a^{2} / b d^{2} ; q^{4}\right]_{k}\left(q^{3} a / b ; q^{3}\right)_{k}\left(b d / q^{4} a ; q^{-1}\right)_{k}} q^{k}, \\
& \Delta \mathrm{B}_{k}=-\frac{\left(1-q^{4+5 k} a\right)\left(1-q^{3+k} a / b d\right)\left(1-q^{2+2 k} b\right)\left(1-q^{9} a^{2} / b^{2} d^{3}\right)}{\left(1-q^{5} a / b d^{2}\right)\left(1-q^{7} a^{2} / b^{2} d^{2}\right)\left(1-q^{6} a / d\right)(1-b d)} \\
& \times\left[\begin{array}{c}
q^{4} a / b d \\
b d^{2} / q^{4} a
\end{array} \mid q\right]_{k}\left[\begin{array}{c}
d / q^{2} \\
q^{9} a^{2} / b^{2} d^{2}
\end{array} \mid q^{2}\right]_{k}\left[\begin{array}{c}
b^{2} d^{2} / q^{3} a \\
q^{9} a / d
\end{array} \mid q^{3}\right]_{k}\left[\begin{array}{c}
q^{9} a^{2} / b d^{2} \\
q^{4} b d
\end{array} \mid q^{4}\right]_{k} q^{k} .
\end{aligned}
$$

Then applying the modified Abel lemma on summation by parts, the $U$-sum can alternatively be reformulated as follows:

$$
U_{n}(a, b, d) \frac{\left(1-q^{2} / d\right)\left(1-q^{3} a / d\right)\left(1-q^{5} a^{2} / b^{2} d^{2}\right)}{\left(1-q^{2} a / b d\right)\left(1-q^{5} a / b d^{2}\right)(1-b)\left(1-q^{3} a^{2} / b d\right)}
$$




$$
=\sum_{k=0}^{n-1} \mathrm{~B}_{k} \nabla \mathrm{A}_{k}=\varpi\{\mathcal{R}-1\}-\sum_{k=0}^{n-1} \mathrm{~A}_{k} \Delta \mathrm{B}_{k} .
$$

Observing that the last partial sum results in

$$
-\sum_{k=0}^{n-1} \mathrm{~A}_{k} \Delta \mathrm{B}_{k}=\frac{U_{n}\left(q^{4} a, q^{4} b, d / q^{2}\right)\left(1-q^{2} b\right)\left(1-q^{3} a / b d\right)\left(1-q^{9} a^{2} / b^{2} d^{3}\right)}{(1-b d)\left(1-q^{6} a / d\right)\left(1-q^{5} a / b d^{2}\right)\left(1-q^{7} a^{2} / b^{2} d^{2}\right)},
$$

we derive after some simplification the relation

$$
\begin{aligned}
U_{n}(a, b, d)= & U_{n}\left(q^{4} a, q^{4} b, d / q^{2}\right) \frac{\left(b ; q^{2}\right)_{2}\left(q^{2} a / b d ; q\right)_{2}\left(1-q^{3} a^{2} / b d\right)\left(1-q^{9} a^{2} / b^{2} d^{3}\right)}{\left(q^{5} a^{2} / b^{2} d^{2} ; q^{2}\right)_{2}\left(q^{3} a / d ; q^{3}\right)_{2}\left(1-q^{2} / d\right)(1-b d)} \\
& +\{1-\mathcal{R}(a, b, d)\} \frac{\left(1-b d / q^{2}\right)\left(1-q^{5} a^{2} / b d^{2}\right)(1-a / b)\left(1-q^{3} a / b d\right)}{\left(1-d / q^{2}\right)\left(1-q^{3} a / d\right)\left(1-q^{5} a^{2} / b^{2} d^{2}\right)}
\end{aligned}
$$

Iterating it $m$-times, we get the following expression

$$
\begin{aligned}
U_{n}(a, b, d)= & U_{n}\left(q^{4 m} a, q^{4 m} b, q^{-2 m} d\right) \frac{\left(b ; q^{2}\right)_{2 m}\left(q^{2} a / b d ; q\right)_{2 m}\left[q^{3} a^{2} / b d, q^{9} a^{2} / b^{2} d^{3} ; q^{6}\right]_{m}}{\left(q^{5} a^{2} / b^{2} d^{2} ; q^{2}\right)_{2 m}\left(q^{3} a / d ; q^{3}\right)_{2 m}\left[q^{2} / d, b d ; q^{2}\right]_{m}} \\
& +\frac{\left(1-b d / q^{2}\right)\left(1-q^{3} a / b d\right)(1-a / b)}{\left(1-d / q^{2}\right)\left(1-q^{3} a / d\right)\left(1-q^{5} a^{2} / b^{2} d^{2}\right)} \sum_{k=0}^{m-1} \frac{\left(1-q^{5+8 k} a^{2} / b d^{2}\right)\left(b ; q^{2}\right)_{2 k}}{\left(q^{7} a^{2} / b^{2} d^{2} ; q^{2}\right)_{2 k}} \\
& \times\left\{1-\mathcal{R}\left(q^{4 k} a, q^{4 k} b, q^{-2 k} d\right)\right\}\left[\begin{array}{c}
q^{2} a / b d, q^{5} a / b d \\
q^{4} / d, b d / q^{2}
\end{array} \mid q^{2}\right]_{k}\left[\begin{array}{c}
q^{3} a^{2} / b d, q^{9} a^{2} / b^{2} d^{3} \\
q^{6} a / d, q^{9} a / d
\end{array} \mid q^{6}\right]_{k} q^{2 k}
\end{aligned}
$$

Rewriting the $\mathcal{R}$-function explicitly as

$$
\begin{aligned}
\mathcal{R}\left(q^{4 k} a, q^{4 k} b, q^{-2 k} d\right)= & \frac{1-q^{5+4 n+8 k} a^{2} / b d^{2}}{1-q^{5+8 k} a^{2} / b d^{2}}\left[\begin{array}{c}
q^{4 k} b, q^{-2-2 k} d \\
q^{7+4 k} a^{2} / b^{2} d^{2}
\end{array} \mid q^{2}\right]_{n} \\
& \times \frac{\left[q^{2+2 k} a / b d, q^{4+2 k} a / b d ; q\right]_{n}}{\left(q^{2 k-2} b d ; q^{2}\right)_{2 n}}\left[\begin{array}{c}
b^{2} d^{2} / q^{3} a \\
a / b, q^{6+6 k} a / d
\end{array} \mid q^{3}\right]_{n} \frac{\left(q^{3+6 k} a^{2} / b d ; q^{6}\right)_{n}}{\left(q^{-3-2 k} b d / a ; q^{-1}\right)_{n}} \\
= & \frac{\left[q^{2} a / b d, q^{4} a / b d ; q\right]_{n}}{\left(b d / q^{2} ; q^{2}\right)_{2 n}}\left[\begin{array}{c}
b, d / q^{2} \\
q^{7} a^{2} / b^{2} d^{2}
\end{array} \mid q^{2}\right]_{n}\left[\begin{array}{c}
b^{2} d^{2} / q^{3} a \\
a / b, q^{6} a / d
\end{array} \mid q^{3}\right]_{n} \frac{\left(q^{3} a^{2} / b d ; q^{6}\right)_{n}}{\left(b d / q^{3} a ; q^{-1}\right)_{n}} \\
& \times \frac{1-q^{5+4 n+8 k} a^{2} / b d^{2}}{1-q^{5+8 k} a^{2} / b d^{2}}\left[\begin{array}{c}
q^{2+n} a / b d, q^{5+n} a / b d, q^{4} / d, q^{-2} b d \\
q^{2} a / b d, q^{5} a / b d, q^{4-2 n} / d, q^{4 n-2} b d
\end{array} \mid q^{2}\right]_{k} \\
& \times \frac{\left(q^{6} a / d ; q^{3}\right)_{2 k}}{\left(q^{6+3 n} a / d ; q^{3}\right)_{2 k}}\left[\begin{array}{c}
q^{2 n} b, q^{7} a^{2} / b^{2} d^{2} \\
b, q^{7+2 n} a^{2} / b^{2} d^{2}
\end{array} \mid q^{2}\right]_{2 k} \frac{\left(q^{3+6 n} a^{2} / b d ; q^{6}\right)_{k}}{\left(q^{3} a^{2} / b d ; q^{6}\right)_{k}}
\end{aligned}
$$

and defining further the finite cubic sum in base $q^{2}$ by

$$
\begin{aligned}
U_{m}^{\triangleright}(a, b, d)= & \sum_{k=0}^{m-1}\left(1-q^{5+8 k} a^{2} / b d^{2}\right)\left[\begin{array}{c}
q^{3} a^{2} / b d, q^{9} a^{2} / b^{2} d^{3} \\
q^{6} a / d, q^{9} a / d
\end{array} \mid q^{6}\right]_{k} \\
& \times \frac{\left(b ; q^{2}\right)_{2 k}}{\left(q^{7} a^{2} / b^{2} d^{2} ; q^{2}\right)_{2 k}}\left[\left.\begin{array}{c}
q^{2} a / b d, q^{5} a / b d \\
q^{4} / d, b d / q^{2}
\end{array}\right|^{2}\right]_{k} q^{2 k},
\end{aligned}
$$

we find the following transformation formula.

\section{Theorem 4 (Transformation between quartic and cubic series).}

$$
U_{n}(a, b, d)-U_{n}\left(q^{4 m} a, q^{4 m} b, q^{-2 m} d\right) \frac{\left(b ; q^{2}\right)_{2 m}\left(q^{2} a / b d ; q\right)_{2 m}\left[q^{3} a^{2} / b d, q^{9} a^{2} / b^{2} d^{3} ; q^{6}\right]_{m}}{\left(q^{5} a^{2} / b^{2} d^{2} ; q^{2}\right)_{2 m}\left(q^{3} a / d ; q^{3}\right)_{2 m}\left[q^{2} / d, b d ; q^{2}\right]_{m}}
$$




$$
\begin{aligned}
= & \frac{\left(1-b d / q^{2}\right)\left(1-q^{3} a / b d\right)(1-a / b)}{\left(1-d / q^{2}\right)\left(1-q^{3} a / d\right)\left(1-q^{5} a^{2} / b^{2} d^{2}\right)}\left\{U_{m}^{\triangle}(a, b, d)-U_{m}^{\triangle}\left(q^{5 n} a, q^{2 n} b, q^{2 n} d\right)\right. \\
& \left.\times \frac{\left[q^{2} a / b d, q^{4} a / b d ; q\right]_{n}}{\left(b d / q^{2} ; q^{2}\right)_{2 n}}\left[\begin{array}{c}
b, d / q^{2} \\
q^{7} a^{2} / b^{2} d^{2}
\end{array} \mid q^{2}\right]_{n}\left[\begin{array}{c}
b^{2} d^{2} / q^{3} a \\
a / b, q^{6} a / d
\end{array} \mid q^{3}\right]_{n} \frac{\left(q^{3} a^{2} / b d ; q^{6}\right)_{n}}{\left(b d / q^{3} a ; q^{-1}\right)_{n}}\right\} .
\end{aligned}
$$

By means of the Weierstrass $M$-test, we can compute the limit

$$
\lim _{m, n \rightarrow \infty} U_{n}\left(q^{4 m} a, q^{4 m} b, q^{-2 m} d\right)=\sum_{k=0}^{\infty}\left(\frac{a}{b}\right)^{k} \frac{\left(b^{2} d^{2} / q^{3} a ; q^{3}\right)_{k}}{\left(q^{3} a / b ; q^{3}\right)_{k}} q^{3\left(\begin{array}{c}
k+1 \\
2
\end{array}\right) .}
$$

The limiting case $m, n \rightarrow \infty$ of Theorem 4 leads to the transformation formula.

\section{Proposition 5 (Nonterminating series transformation).}

$$
\begin{aligned}
U(a, b, d) & =\frac{\left(1-b d / q^{2}\right)\left(1-q^{3} a / b d\right)(1-a / b)}{\left(1-d / q^{2}\right)\left(1-q^{3} a / d\right)\left(1-q^{5} a^{2} / b^{2} d^{2}\right)} U^{\triangle}(a, b, d) \\
& \left.+\frac{\left(q^{2} a / b d ; q\right)_{\infty}\left(b ; q^{2}\right)_{\infty}\left[q^{3} a^{2} / b d, q^{9} a^{2} / b^{2} d^{3} ; q^{6}\right]_{\infty}}{\left(q^{3} a / d ; q^{3}\right)_{\infty}\left[q^{2} / d, b d, q^{5} a^{2} / b^{2} d^{2} ; q^{2}\right]_{\infty}} \sum_{k=0}^{\infty}\left(\frac{q^{3} a}{b}\right)^{k} \frac{\left(b^{2} d^{2} / q^{3} a ; q^{3}\right)_{k}}{\left(q^{3} a / b ; q^{3}\right)_{k}} q^{3} \begin{array}{c}
k \\
2
\end{array}\right) .
\end{aligned}
$$

When $b=a$, this proposition reduces to the following relation

$$
U(a, a, d)=\frac{\left(q^{2} / d ; q\right)_{\infty}\left(a ; q^{2}\right)_{\infty}\left[q^{3} a / d, q^{9} / d^{3} ; q^{6}\right]_{\infty}}{\left(q^{3} a / d ; q^{3}\right)_{\infty}\left[q^{2} / d, a d, q^{5} / d^{2} ; q^{2}\right]_{\infty}} \sum_{k=0}^{\infty} \frac{\left(a d^{2} / q^{3} ; q^{3}\right)_{k}}{\left(q^{3} ; q^{3}\right)_{k}} q^{3\left(\begin{array}{c}
k \\
2
\end{array}\right)+3 k}
$$

Taking $d=1$ in the last equation and noting $U(a, b, 1)=1-a$, we get

$$
\sum_{k=0}^{\infty} \frac{\left(a / q^{3} ; q^{3}\right)_{k}}{\left(q^{3} ; q^{3}\right)_{k}} q^{3\left(\begin{array}{c}
k \\
2
\end{array}\right)+3 k}=\frac{\left(a ; q^{6}\right)_{\infty}}{\left(q^{3} ; q^{6}\right)_{\infty}}
$$

which results also from a limiting case of the $q$-Bailey-Daum formula (cf. [12, II-9]).

Combining the last two equations leads us to the nonterminating series identity.

\section{Corollary 6 (Gasper-Rahman [12, Exercise 3.29(i)]).}

$$
\begin{gathered}
\sum_{k=0}^{\infty} \frac{1-q^{5 k} a}{1-a}\left[\begin{array}{c}
a, d \\
q^{5} / d^{2}
\end{array} \mid q^{2}\right]_{k}\left[\begin{array}{c}
a d^{2} / q^{3} \\
q^{3}, q^{3} a / d
\end{array} \mid q^{3}\right]_{k} \frac{\left(q^{2} / d ; q\right)_{k}\left(q^{3} a / d ; q^{6}\right)_{k}}{\left(a d ; q^{2}\right)_{2 k}} q^{\left(\begin{array}{c}
k \\
2
\end{array}\right)\left(-q^{3} / d\right)^{k}} \\
=\left[\begin{array}{c}
q^{2} a, q^{3} / d \\
a d, q^{5} / d^{2}
\end{array} \mid q^{2}\right]_{\infty}\left[\begin{array}{c}
a d^{2}, q^{9} / d^{3} \\
q^{3}, q^{6} a / d
\end{array} \mid q^{6}\right]_{\infty} .
\end{gathered}
$$

\subsection{Quartic series to quartic series}

Finally, for the two sequences given by

$$
\begin{aligned}
\mathrm{A}_{k} & =\frac{\left(q^{3} a / b d ; q\right)_{k}\left(q^{2} b ; q^{2}\right)_{k}\left(b^{2} d^{2} / a ; q^{3}\right)_{k}\left(q^{5} a^{2} / b^{2} d ; q^{4}\right)_{k}}{\left(q^{2} b d ; q^{4}\right)_{k}\left(q^{3} a / b ; q^{3}\right)_{k}\left(q^{5} a^{2} / b^{2} d^{2} ; q^{2}\right)_{k}\left(b^{2} d / a ; q\right)_{k}} \\
\mathrm{~B}_{k} & =\frac{\left[q a / b d, b^{2} d / a ; q\right]_{k}\left(d / q^{2} ; q^{2}\right)_{k}\left(q^{3} a^{2} / b d ; q^{6}\right)_{k}}{\left[b d, q a^{2} / b^{2} d ; q^{4}\right]_{k}\left(q^{3} a / d ; q^{3}\right)_{k}\left(b d / q^{2} a ; q^{-1}\right)_{k}}
\end{aligned}
$$

we have no difficulty to check the relations

$$
\varpi:=\mathrm{A}_{-1} \mathrm{~B}_{0}=\frac{\left(1-b^{2} d / q a\right)\left(1-b^{2} d^{2} / q^{3} a^{2}\right)(1-b / a)\left(1-b d / q^{2}\right)}{\left(1-b d / q^{2} a\right)(1-b)\left(1-b^{2} d^{2} / q^{3} a\right)\left(1-b^{2} d / q a^{2}\right)},
$$




$$
\begin{aligned}
\mathcal{R}:= & \frac{\mathrm{A}_{n-1} \mathrm{~B}_{n}}{\mathrm{~A}_{-1} \mathrm{~B}_{0}}=\frac{1-q^{n-1} b^{2} d / a}{1-q^{-1} b^{2} d / a}\left[\begin{array}{c}
b, d / q^{2} \\
q^{3} a^{2} / b^{2} d^{2}
\end{array} \mid q^{2}\right]_{n} \\
& \times \frac{\left[q a / b d, q^{2} a / b d ; q\right]_{n}}{\left(b d / q^{2} ; q^{2}\right)_{2 n}}\left[\begin{array}{c}
b^{2} d^{2} / q^{3} a \\
a / b, q^{3} a / d
\end{array} \mid q^{3}\right]_{n} \frac{\left(q^{3} a^{2} / b d ; q^{6}\right)_{n}}{\left(b d / q^{2} a ; q^{-1}\right)_{n}}
\end{aligned}
$$

and compute the finite differences

$$
\begin{aligned}
& \nabla \mathrm{A}_{k}=\frac{\left(1-q^{5 k} a\right)\left(1-q^{2-2 k} / d\right)\left(1-q^{1+k} a / b d\right)\left(1-q^{3} a^{2} / b^{3} d^{2}\right)}{(1-b)\left(1-q^{2} a / b d\right)\left(1-q a^{2} / b^{2} d\right)\left(1-q^{3} a / b^{2} d^{2}\right)} \\
& \times\left[\begin{array}{c}
q^{2} a / b d \\
b^{2} d / a
\end{array} \mid q\right]_{k}\left[\begin{array}{c}
b \\
q^{5} a^{2} / b^{2} d^{2}
\end{array} \mid q^{2}\right]_{k}\left[\begin{array}{c}
b^{2} d^{2} / q^{3} a \\
q^{3} a / b
\end{array} \mid q^{3}\right]_{k}\left[\begin{array}{c}
q a^{2} / b^{2} d \\
q^{2} b d
\end{array} \mid q^{4}\right]_{k} q^{3 k}, \\
& \Delta \mathrm{B}_{k}=-\frac{\left(1-q^{1+5 k} a\right)\left(1-q^{3 k} a / b\right)\left(1-q^{2+2 k} b\right)\left(1-q^{3+2 k} a^{2} / b^{2} d^{2}\right)}{(1-b d)\left(1-q a^{2} / b^{2} d\right)\left(1-q^{3} a / d\right)\left(1-q^{2} a / b d\right)} \\
& \times \frac{\left[q a / b d, b^{2} d / a ; q\right]_{k}\left(d / q^{2} ; q^{2}\right)_{k}\left(q^{3} a^{2} / b d ; q^{6}\right)_{k}}{\left[q^{4} b d, q^{5} a^{2} / b^{2} d ; q^{4}\right]_{k}\left(q^{6} a / d ; q^{3}\right)_{k}\left(b d / q^{3} a ; q^{-1}\right)_{k}} q^{-k} .
\end{aligned}
$$

Then by means of the modified Abel lemma on summation by parts, the $U$-sum can be reformulated as follows:

$$
\begin{gathered}
U_{n}(a, b, d) \frac{\left(1-q^{2} / d\right)(1-q a / b d)\left(1-q^{3} a^{2} / b^{3} d^{2}\right)}{(1-b)\left(1-q^{2} a / b d\right)\left(1-q a^{2} / b^{2} d\right)\left(1-q^{3} a / b^{2} d^{2}\right)} \\
\quad=\sum_{k=0}^{n-1} \mathrm{~B}_{k} \nabla \mathrm{A}_{k}=\varpi\{\mathcal{R}-1\}-\sum_{k=0}^{n-1} \mathrm{~A}_{k} \Delta \mathrm{B}_{k} .
\end{gathered}
$$

Writing the last partial sum in terms of $U$-sum as

$$
-\sum_{k=0}^{n-1} \mathrm{~A}_{k} \Delta \mathrm{B}_{k}=\frac{U_{n}\left(q a, q^{4} b, d / q^{2}\right)(1-b / a)\left(1-q^{2} b\right)\left(1-b^{2} d^{2} / q^{3} a^{2}\right)}{(1-b d)\left(1-b^{2} d / q a^{2}\right)\left(1-q^{3} a / d\right)\left(1-b d / q^{2} a\right)}
$$

we derive after some simplification the following relation

$$
\begin{aligned}
U_{n}(a, b, d)= & \{1-\mathcal{R}(a, b, d)\} \frac{(1-a / b)\left(1-b d / q^{2}\right)\left(1-b^{2} d / q a\right)\left(1-b^{2} d^{2} / q^{3} a^{2}\right)}{\left(1-d / q^{2}\right)(1-b d / q a)\left(1-b^{3} d^{2} / q^{3} a^{2}\right)} \\
& -U_{n}\left(q a, q^{4} b, d / q^{2}\right) \frac{\left(q^{2} a / b d\right)\left(b ; q^{2}\right)_{2}\left(1-b^{2} d^{2} / q^{3} a\right)\left(1-b^{2} d^{2} / q^{3} a^{2}\right)(1-b / a)}{(1-b d)\left(1-q^{2} / d\right)(1-b d / q a)\left(1-q^{3} a / d\right)\left(1-b^{3} d^{2} / q^{3} a^{2}\right)} .
\end{aligned}
$$

Iterating it $m$-times, we get the following expression

$$
\begin{aligned}
& U_{n}(a, b, d)=U_{n}\left(q^{m} a, q^{4 m} b, q^{-2 m} d\right)\left[\begin{array}{c}
b^{2} d^{2} / q^{3} a^{2} \\
q^{2} / d, b d
\end{array} \mid q^{2}\right]_{m} \\
& \times \frac{\left(b ; q^{2}\right)_{2 m}}{\left[b d / q a, b d / q^{2} a ; q\right]_{m}}\left[\begin{array}{c}
b / a, b^{2} d^{2} / q^{3} a \\
q^{3} a / d
\end{array} \mid q^{3}\right]_{m} \frac{\left(q^{2} a / b d ; q^{-1}\right)_{m}}{\left(b^{3} d^{2} / q^{3} a^{2} ; q^{6}\right)_{m}} \\
& +\frac{(1-a / b)\left(1-b d / q^{2}\right)\left(1-b^{2} d^{2} / q^{3} a^{2}\right)}{\left(1-d / q^{2}\right)(1-b d / q a)\left(1-b^{3} d^{2} / q^{3} a^{2}\right)} \sum_{k=0}^{m-1}\left(1-q^{5 k-1} b^{2} d / a\right)\left[\begin{array}{c}
b^{2} d^{2} / q a^{2} \\
q^{4} / d, b d / q^{2}
\end{array} \mid q^{2}\right]_{k} \\
& \times\left\{1-\mathcal{R}\left(q^{k} a, q^{4 k} b, q^{-2 k} d\right)\right\} \frac{\left(b ; q^{2}\right)_{2 k}}{(b d / a ; q)_{k}}\left[\begin{array}{c}
q^{3} b / a, b^{2} d^{2} / q^{3} a \\
q^{3} a / d
\end{array} \mid q^{3}\right]_{k} \frac{(q a / b d)^{k} q^{-\left(\begin{array}{c}
k \\
2
\end{array}\right)}}{\left(q^{3} b^{3} d^{2} / a^{2} ; q^{6}\right)_{k}} .
\end{aligned}
$$

Separating $k$ and $n$ factorials in the $\mathcal{R}$-function

$$
\mathcal{R}\left(q^{k} a, q^{4 k} b, q^{-2 k} d\right)=\frac{1-q^{n+5 k-1} b^{2} d / a}{1-q^{5 k-1} b^{2} d / a}\left[\begin{array}{c}
q^{4 k} b, q^{-2-2 k} d \\
q^{3-2 k} a^{2} / b^{2} d^{2}
\end{array} \mid q^{2}\right]_{n}
$$




$$
\begin{aligned}
& \times \frac{\left[q^{1-k} a / b d, q^{2-k} a / b d ; q\right]_{n}}{\left(q^{2 k-2} b d ; q^{2}\right)_{2 n}}\left[\begin{array}{c}
q^{3 k-3} b^{2} d^{2} / a \\
q^{-3 k} a / b, q^{3+3 k} a / d
\end{array} \mid q^{3}\right]_{n} \frac{\left(q^{3} a^{2} / b d ; q^{6}\right)_{n}}{\left(q^{k-2} b d / a ; q^{-1}\right)_{n}} \\
= & \frac{\left[q a / b d, q^{2} a / b d ; q\right]_{n}}{\left(b d / q^{2} ; q^{2}\right)_{2 n}}\left[\begin{array}{c}
b, d / q^{2} \\
q^{3} a^{2} / b^{2} d^{2}
\end{array} \mid q^{2}\right]_{n}\left[\begin{array}{c}
b^{2} d^{2} / q^{3} a \\
a / b, q^{3} a / d
\end{array} \mid q^{3}\right]_{n} \frac{\left(q^{3} a^{2} / b d ; q^{6}\right)_{n}}{\left(b d / q^{2} a ; q^{-1}\right)_{n}} \\
& \times \frac{1-q^{n+5 k-1} b^{2} d / a}{1-q^{5 k-1} b^{2} d / a}\left[\begin{array}{c}
q^{3 n-3} b^{2} d^{2} / a, q^{3-3 n} b / a, q^{3} a / d \\
b^{2} d^{2} / q^{3} a, q^{3} b / a, q^{3+3 n} a / d
\end{array} \mid q^{3}\right]_{k} q^{n k} \\
& \times \frac{(b d / a ; q)_{k}\left(q^{2 n} b ; q^{2}\right)_{2 k}}{\left(q^{-n} b d / a ; q\right)_{k}\left(b ; q^{2}\right)_{2 k}}\left[\begin{array}{c}
q^{4} / d, b d / q^{2}, q^{-1-2 n} b^{2} d^{2} / a^{2} \\
q^{4-2 n} / d, q^{4 n-2} b d, b^{2} d^{2} / q a^{2}
\end{array} \mid q^{2}\right]_{k},
\end{aligned}
$$

and then defining the partial sum of quartic series

$$
\begin{aligned}
U_{m}^{\star}(a, b, d)= & \sum_{k=0}^{m-1}\left(1-q^{5 k-1} b^{2} d / a\right)\left[\begin{array}{c}
b^{2} d^{2} / q a^{2} \\
q^{4} / d, b d / q^{2}
\end{array} \mid q^{2}\right]_{k} \frac{\left(b ; q^{2}\right)_{2 k}}{(b d / a ; q)_{k}} \\
& \times\left[\begin{array}{c}
q^{3} b / a, b^{2} d^{2} / q^{3} a \\
q^{3} a / d
\end{array} \mid q^{3}\right]_{k} \frac{(-q a / b d)^{k} q^{-\left(\begin{array}{c}
k \\
2
\end{array}\right)}}{\left(q^{3} b^{3} d^{2} / a^{2} ; q^{6}\right)_{k}},
\end{aligned}
$$

we establish the following transformation formula.

\section{Theorem 7 (Transformation between two quartic series).}

$$
\begin{aligned}
U_{n}(a, b, d)- & U_{n}\left(q^{m} a, q^{4 m} b, q^{-2 m} d\right)\left[\begin{array}{c}
b^{2} d^{2} / q^{3} a^{2} \\
b d, q^{2} / d
\end{array} \mid q^{2}\right]_{m} \\
& \times \frac{\left(b ; q^{2}\right)_{2 m}}{\left[b d / q a, b d / q^{2} a ; q\right]_{m}}\left[\begin{array}{c}
b^{2} d^{2} / q^{3} a, b / a \\
q^{3} a / d
\end{array} \mid q^{3}\right]_{m} \frac{\left(q^{2} a / b d ; q^{-1}\right)_{m}}{\left(b^{3} d^{2} / q^{3} a^{2} ; q^{6}\right)_{m}} \\
= & \frac{(1-a / b)\left(1-b d / q^{2}\right)\left(1-b^{2} d^{2} / q^{3} a^{2}\right)}{\left(1-d / q^{2}\right)(1-b d / q a)\left(1-b^{3} d^{2} / q^{3} a^{2}\right)}\left\{U_{m}^{\star}(a, b, d)-U_{m}^{\star}\left(q^{5 n} a, q^{2 n} b, q^{2 n} d\right)\right. \\
& \left.\times \frac{\left[q a / b d, q^{2} a / b d ; q\right]_{n}}{\left(b d / q^{2} ; q^{2}\right)_{2 n}}\left[\begin{array}{c}
b, d / q^{2} \\
q^{3} a^{2} / b^{2} d^{2} \mid q^{2}
\end{array}\right]_{n}\left[\begin{array}{c}
b^{2} d^{2} / q^{3} a \\
a / b, q^{3} a / d
\end{array} \mid q^{3}\right]_{n} \frac{\left(q^{3} a^{2} / b d ; q^{6}\right)_{n}}{\left(b d / q^{2} a ; q^{-1}\right)_{n}}\right\} .
\end{aligned}
$$

In particular for $m=n$, we have the reduced transformation.

Proposition 8 (Transformation between two quartic series: $q^{3} a^{2}=b^{2} d^{2}$ ).

$$
\begin{aligned}
U_{n}(a, b, d)= & U_{n}^{\star}\left(q^{5 n} a, q^{2 n} b, q^{2 n} d\right) \frac{\left(a ; q^{3}\right)_{n}\left(b d ; q^{6}\right)_{n}}{\left(q^{3} a / d ; q^{3}\right)_{n}\left(q^{3} a / b ; q^{3}\right)_{n-1}} \\
& \times \frac{(q a / b d ; q)_{n}\left(q^{3} a / b d ; q\right)_{n-1}}{\left(q a / b d ; q^{-1}\right)_{n}\left(b d ; q^{2}\right)_{2 n-1}}\left[\begin{array}{c}
q^{2} b, d \\
q^{2}
\end{array} \mid q^{2}\right]_{n-1} .
\end{aligned}
$$

In order to examine the limiting case $n \rightarrow \infty$ of the last equation, we write $U_{n}^{\star}\left(q^{5 n} a, q^{2 n} b\right.$, $\left.q^{3 / 2+2 n} a / b\right)$ explicitly as follows:

$$
\begin{aligned}
U_{n}^{\star}\left(q^{5 n} a, q^{2 n} b, q^{3 / 2+2 n} a / b\right)= & \sum_{k=0}^{n-1}\left(1-q^{n+5 k+1 / 2} b\right)\left[\begin{array}{c}
q^{2-2 n} \\
q^{5 / 2-2 n} b / a, q^{4 n-1 / 2} a
\end{array} \mid q^{2}\right]_{k} \\
& \times\left[\begin{array}{c}
q^{3-3 n} b / a, q^{3 n} a \\
q^{3 / 2+3 n} b
\end{array} \mid q^{3}\right]_{k} \frac{\left(q^{2 n} b ; q^{2}\right)_{2 k}\left(-q^{n-1 / 2}\right)^{k}}{\left(q^{3 / 2-n} ; q\right)_{k}\left(q^{6} b ; q^{6}\right)_{k}} q^{-\left(\begin{array}{c}
k \\
2
\end{array}\right)}
\end{aligned}
$$

Inverting the summation index $k \rightarrow n-1-k$ and then applying the relation

$$
\frac{\left(q^{2-2 n} ; q^{2}\right)_{n-1-k}\left(q^{3-3 n} b / a ; q^{3}\right)_{n-1-k}}{\left(q^{5 / 2-2 n} b / a ; q^{2}\right)_{n-1-k}\left(q^{3 / 2-n} ; q\right)_{n-1-k}}
$$




$$
=\frac{\left(q^{2} ; q^{2}\right)_{n-1}\left(q^{3} a / b ; q^{3}\right)_{n-1}}{\left(q^{3 / 2} a / b ; q^{2}\right)_{n-1}\left(q^{1 / 2} ; q\right)_{n-1}} q^{1-n^{2}} \frac{\left(q^{1 / 2} ; q\right)_{k}\left(q^{3 / 2} a / b ; q^{2}\right)_{k}}{\left(q^{2} ; q^{2}\right)_{k}\left(q^{3} a / b ; q^{3}\right)_{k}} q^{k^{2}+2 k}
$$

we can reformulate the finite sum $U_{n}^{\star}\left(q^{5 n} a, q^{2 n} b, q^{3 / 2+2 n} a / b\right)$ as

$$
\begin{aligned}
(-1)^{n-1} & \frac{\left(q^{2} ; q^{2}\right)_{n-1}\left(q^{3} a / b ; q^{3}\right)_{n-1}}{\left(q^{3 / 2} a / b ; q^{2}\right)_{n-1}\left(q^{1 / 2} ; q\right)_{n-1}} q^{\frac{1-n^{2}}{2}} \sum_{k=0}^{n-1}(-1)^{k} \frac{\left(q^{1 / 2} ; q\right)_{k}\left(q^{3 / 2} a / b ; q^{2}\right)_{k}}{\left(q^{2} ; q^{2}\right)_{k}\left(q^{3} a / b ; q^{3}\right)_{k}} q^{\frac{k(k+2)}{2}} \\
& \times \frac{\left(1-q^{6 n-5 k-9 / 2} b\right)\left(q^{2 n} b ; q^{2}\right)_{2 n-2-2 k}\left(q^{3 n} a ; q^{3}\right)_{n-1-k}}{\left(q^{4 n-1 / 2} a ; q^{2}\right)_{n-1-k}\left(q^{3 / 2+3 n} b ; q^{3}\right)_{n-1-k}\left(q^{6} b ; q^{6}\right)_{n-1-k}} .
\end{aligned}
$$

Substituting this expression into Proposition 8 and then letting $n \rightarrow \infty$, we derive the following transformation formula

$$
\begin{aligned}
U\left(a, b, q^{3 / 2} a / b\right)= & \frac{\left(b ; q^{2}\right)_{\infty}\left(a ; q^{3}\right)_{\infty}\left(q^{3 / 2} a ; q^{6}\right)_{\infty}}{\left(q^{3 / 2} a ; q^{2}\right)_{\infty}\left(q^{3 / 2} b ; q^{3}\right)_{\infty}\left(b ; q^{6}\right)_{\infty}} \\
& \times \sum_{k=0}^{\infty}\left(-q^{3 / 2}\right)^{k} \frac{\left(q^{1 / 2} ; q\right)_{k}\left(q^{3 / 2} a / b ; q^{2}\right)_{k}}{\left(q^{2} ; q^{2}\right)_{k}\left(q^{3} a / b ; q^{3}\right)_{k}} q^{\left(\begin{array}{c}
k \\
2
\end{array}\right)} .
\end{aligned}
$$

From this transformation, we can derive two new interesting summation formulae. First, taking $b=1$ in this equation and keeping in mind that $U(a, 1, d)=1$, we obtain the following remarkable summation formula.

\section{Corollary 9 (Nonterminating series identity).}

$$
\sum_{k=0}^{\infty}\left(-q^{3 / 2}\right)^{k} \frac{\left(q^{1 / 2} ; q\right)_{k}\left(q^{3 / 2} a ; q^{2}\right)_{k}}{\left(q^{2} ; q^{2}\right)_{k}\left(q^{3} a ; q^{3}\right)_{k}} q^{\left(\begin{array}{c}
k \\
2
\end{array}\right)}=\frac{\left(q^{3 / 2} a ; q^{2}\right)_{\infty}\left(q^{3 / 2} ; q^{3}\right)_{\infty}\left(q^{6} ; q^{6}\right)_{\infty}}{\left(q^{2} ; q^{2}\right)_{\infty}\left(q^{3} a ; q^{3}\right)_{\infty}\left(q^{3 / 2} a ; q^{6}\right)_{\infty}}
$$

The special case $a=0$ of this corollary recovers an identity of Rogers-Ramanujan type due to Stanton [18, p. 61]:

$$
\sum_{k=0}^{\infty} \frac{\left(-q ; q^{2}\right)_{k}}{\left(q^{4} ; q^{4}\right)_{k}} q^{k(k+2)}=\frac{\left(-q ; q^{2}\right)_{\infty}}{\left(q^{2} ; q^{2}\right)_{\infty}}\left[q^{6}, q, q^{5} ; q^{6}\right]_{\infty} .
$$

Combining (1) with Corollary 9 yields another formula for quartic series.

\section{Corollary 10 (Nonterminating series identity).}

$$
\begin{gathered}
\sum_{k=0}^{\infty}(-1)^{k} \frac{1-q^{5 k} a}{1-a}\left[\begin{array}{c}
b, q^{3 / 2} a / b \\
q^{2}
\end{array} \mid q^{2}\right]_{k}\left[\begin{array}{c}
a \\
q^{3} a / b, q^{3 / 2} b
\end{array} \mid q^{3}\right]_{k} \frac{\left(q^{1 / 2} ; q\right)_{k}\left(q^{3 / 2} a ; q^{6}\right)_{k}}{\left(q^{3 / 2} a ; q^{2}\right)_{2 k}} q^{\frac{k^{2}+2 k}{2}} \\
=\left[\begin{array}{c}
b, q^{3 / 2} a / b \\
q^{2}, q^{3 / 2} a
\end{array} \mid q^{2}\right]_{\infty}\left[\begin{array}{c}
q^{3} a, q^{3 / 2} \\
q^{3} a / b, q^{3 / 2} b
\end{array} \mid q^{3}\right]_{\infty}\left[\begin{array}{c}
q^{6}, q^{3 / 2} a \\
b, q^{3 / 2} a / b
\end{array} \mid q^{6}\right]_{\infty} .
\end{gathered}
$$

\section{Transformation and summation formulae for $V_{n}(a, b, d)$}

The quartic series $V_{n}(a, c, e)$ may be considered as dual one to the $U_{n}(a, b, d)$ in the last section in the sense that the numerator factorials and denominator factorials are inverted. This section will be devoted analogously to investigation of summation and transformation formulae for $V_{n}(a, c, e)$. As the series $U_{n}(a, c, e)$, the following expression for $V_{n}(a, c, e)$ makes its well-poised structure more transparent

$$
\begin{aligned}
V_{n}(a, c, e)= & \sum_{k=0}^{n-1}\left(1-q^{5 k} a\right) \frac{\left(q c^{2} e^{2} / a^{2} ; q^{2}\right)_{k}}{\left(q^{2} a^{3} / c^{2} e^{2} ; q^{3}\right)_{k}} \frac{\left[q c, q e ; q^{3}\right]_{k}}{\left[q a / c, q a / e ; q^{2}\right]_{k}} q^{k} \\
& \times \frac{\left[a^{2} / c e, q^{2} a^{2} / c e ; q^{4}\right]_{k}}{[q c e / a, q c e / a ; q]_{k}} \frac{\left(q^{-1} a / c e ; q^{-1}\right)_{k}}{\left(q^{5} c e ; q^{6}\right)_{k}} .
\end{aligned}
$$




\subsection{Quartic series to quadratic series}

Let $\mathrm{A}_{k}$ and $\mathrm{B}_{k}$ be defined by

$$
\begin{aligned}
\mathrm{A}_{k} & =\frac{\left[q^{3} c^{2} e^{2} / a^{2}, a^{4} / q c^{3} e^{3} ; q^{2}\right]_{k}\left[q^{4} c, q^{4} e ; q^{3}\right]_{k}}{\left[q^{2} a^{3} / c^{2} e^{2}, q^{6} c^{3} e^{3} / a^{3} ; q^{3}\right]_{k}\left[q a / c, q a / e ; q^{2}\right]_{k}}, \\
\mathrm{~B}_{k} & =\frac{\left(q^{6} c^{3} e^{3} / a^{3} ; q^{3}\right)_{k}\left(a^{2} / c e ; q^{2}\right)_{2 k}\left(a / q^{2} c e ; q^{-1}\right)_{k}}{\left(a^{4} / q^{3} c^{3} e^{3} ; q^{2}\right)_{k}\left[q c e / a, q^{3} c e / a ; q\right]_{k}\left(q^{5} c e ; q^{6}\right)_{k}} .
\end{aligned}
$$

We can easily show the following relations

$$
\begin{aligned}
\varpi:= & \mathrm{A}_{-1} \mathrm{~B}_{0}=\frac{(1-a / q c)(1-a / q e)\left(1-a^{3} / q c^{2} e^{2}\right)\left(1-q^{3} c^{3} e^{3} / a^{3}\right)}{(1-q c)(1-q e)\left(1-q c^{2} e^{2} / a^{2}\right)\left(1-a^{4} / q^{3} c^{3} e^{3}\right)}, \\
\mathcal{R}:= & \frac{\mathrm{A}_{n-1} \mathcal{B}_{n}}{\mathrm{~A}_{-1} \mathcal{B}_{0}}=\frac{1-q^{3+3 n} c^{3} e^{3} / a^{3}}{1-q^{3} c^{3} e^{3} / a^{3}}\left[\begin{array}{c}
q c^{2} e^{2} / a^{2} \\
a / q c, a / q e
\end{array} \mid q^{2}\right]_{n} \\
& \times \frac{\left(a^{2} / c e ; q^{2}\right)_{2 n}}{\left[q c e / a, q^{3} c e / a ; q\right]_{n}}\left[\begin{array}{c}
q c, q e \\
a^{3} / q c^{2} e^{2}
\end{array} \mid q^{3}\right]_{n} \frac{\left(a / q^{2} c e ; q^{-1}\right)_{n}}{\left(q^{5} c e ; q^{6}\right)_{n}} ;
\end{aligned}
$$

and calculate the finite differences

$$
\begin{aligned}
\nabla \mathrm{A}_{k}= & \frac{\left(1-q^{5 k} a\right)\left(1-q^{2+k} c e / a\right)\left(1-q^{2} c^{2} e^{3} / a^{3}\right)\left(1-q^{2} c^{3} e^{2} / a^{3}\right)}{(1-q c)(1-q e)\left(1-q c^{2} e^{2} / a^{2}\right)\left(1-q^{3} c^{3} e^{3} / a^{4}\right)} \\
& \times\left[\begin{array}{c}
q c^{2} e^{2} / a^{2}, a^{4} / q^{3} c^{3} e^{3} \\
q a / c, \quad q a / e
\end{array} \mid q^{2}\right]_{k}\left[\begin{array}{c}
q c, q q \\
q^{2} a^{3} / c^{2} e^{2}, q^{6} c^{3} e^{3} / a^{3}
\end{array} \mid q^{3}\right]_{k} q^{2 k}, \\
\Delta \mathrm{B}_{k}= & -\frac{\left(1-q^{3+5 k} a\right)\left(1-q^{1-3 k} c^{2} e^{2} / a^{3}\right)\left(1-q^{3+2 k} c^{2} e^{2} / a^{2}\right)\left(1-q^{5+2 k} c^{2} e^{2} / a^{2}\right)}{\left(1-q^{3} c^{3} e^{3} / a^{4}\right)(1-q c e / a)\left(1-q^{3} c e / a\right)\left(1-q^{5} c e\right)} \\
& \times \frac{\left(q^{6} c^{3} e^{3} / a^{3} ; q^{3}\right)_{k}\left(a^{2} / c e ; q^{2}\right)_{2 k}\left(a / q^{2} c e ; q^{-1}\right)_{k}}{\left(a^{4} / q c^{3} e^{3} ; q^{2}\right)_{k}\left[q^{2} c e / a, q^{4} c e / a ; q\right]_{k}\left(q^{11} c e ; q^{6}\right)_{k}} q^{2 k} .
\end{aligned}
$$

Applying the modified Abel lemma on summation by parts, we can manipulate the finite $V$-sum as follows:

$$
\begin{aligned}
& V_{n}(a, c, e) \frac{\left(1-q^{2} c e / a\right)\left(1-q^{2} c^{2} e^{3} / a^{3}\right)\left(1-q^{2} c^{3} e^{2} / a^{3}\right)}{(1-q c)(1-q e)\left(1-q c^{2} e^{2} / a^{2}\right)\left(1-q^{3} c^{3} e^{3} / a^{4}\right)} \\
&=\sum_{k=0}^{n-1} \mathrm{~B}_{k} \nabla \mathrm{A}_{k}=\varpi\{\mathcal{R}-1\}-\sum_{k=0}^{n-1} \mathrm{~A}_{k} \Delta \mathrm{B}_{k} .
\end{aligned}
$$

Noting that the last partial sum results in

$$
-\sum_{k=0}^{n-1} \mathrm{~A}_{k} \Delta \mathrm{B}_{k}=V_{n}\left(q^{3} a, q^{3} c, q^{3} e\right) \frac{\left(1-q c^{2} e^{2} / a^{3}\right)\left(1-q^{3} c^{2} e^{2} / a^{2}\right)\left(1-q^{5} c^{2} e^{2} / a^{2}\right)}{\left(1-q^{3} c^{3} e^{3} / a^{4}\right)(1-q c e / a)\left(1-q^{3} c e / a\right)\left(1-q^{5} c e\right)}
$$

we derive after some simplification the recurrence relation

$$
\begin{aligned}
V_{n}(a, c, e)= & V_{n}\left(q^{3} a, q^{3} c, q^{3} e\right) \frac{(1-q c)(1-q e)\left(1-q c^{2} e^{2} / a^{3}\right)\left(q c^{2} e^{2} / a^{2} ; q^{2}\right)_{3}}{\left(1-q^{5} c e\right)\left(1-q^{2} c^{2} e^{3} / a^{3}\right)\left(1-q^{2} c^{3} e^{2} / a^{3}\right)(q c e / a ; q)_{3}} \\
& -\{1-\mathcal{R}(a, c, e)\} \frac{a(1-q c / a)(1-q e / a)\left(1-q c^{2} e^{2} / a^{3}\right)\left(1-q^{3} c^{3} e^{3} / a^{3}\right)}{\left(1-q^{2} c e / a\right)\left(1-q^{2} c^{3} e^{2} / a^{3}\right)\left(1-q^{2} c^{2} e^{3} / a^{3}\right)} .
\end{aligned}
$$

Iterating it $m$-times, we get the following expression

$$
V_{n}(a, c, e)=V_{n}\left(q^{3 m} a, q^{3 m} c, q^{3 m} e\right) \frac{\left[q c, q e, q c^{2} e^{2} / a^{3} ; q^{3}\right]_{m}\left(q c^{2} e^{2} / a^{2} ; q^{2}\right)_{3 m}}{\left[q^{5} c e, q^{2} c^{2} e^{3} / a^{3}, q^{2} c^{3} e^{2} / a^{3} ; q^{6}\right]_{m}(q c e / a ; q)_{3 m}}
$$




$$
\begin{aligned}
& -\frac{a(1-q c / a)(1-q e / a)\left(1-q c^{2} e^{2} / a^{3}\right)}{\left(1-q^{2} c e / a\right)\left(1-q^{2} c^{2} e^{3} / a^{3}\right)\left(1-q^{2} c^{3} e^{2} / a^{3}\right)} \\
& \times \sum_{k=0}^{m-1}\left(1-q^{3+9 k} c^{3} e^{3} / a^{3}\right)\left[\begin{array}{c}
q c, q e, q^{4} c^{2} e^{2} / a^{3} \\
q c e / a, q^{3} c e / a, q^{5} c e / a
\end{array} \mid q^{3}\right]_{k} q^{3 k} \\
& \times\left\{1-\mathcal{R}\left(q^{3 k} a, q^{3 k} c, q^{3 k} e\right)\right\}\left[\begin{array}{c}
q c^{2} e^{2} / a^{2}, q^{3} c^{2} e^{2} / a^{2}, q^{5} c^{2} e^{2} / a^{2} \\
q^{5} c e, q^{8} c^{2} e^{3} / a^{3}, q^{8} c^{3} e^{2} / a^{3}
\end{array} \mid q^{6}\right]_{k} .
\end{aligned}
$$

Writing explicitly the $\mathcal{R}$-function by separating $k$ and $n$ factorials

$$
\begin{aligned}
\mathcal{R}\left(q^{3 k} a, q^{3 k} c, q^{3 k} e\right)= & \frac{1-q^{3+3 n+9 k} c^{3} e^{3} / a^{3}}{1-q^{3+9 k} c^{3} e^{3} / a^{3}}\left[\begin{array}{c}
q^{1+6 k} c^{2} e^{2} / a^{2} \\
a / q c, a / q e
\end{array} \mid q^{2}\right]_{n} \\
& \times \frac{\left(a^{2} / c e ; q^{2}\right)_{2 n}}{\left[q^{1+3 k} c e / a, q^{3+3 k} c e / a ; q\right]_{n}}\left[\begin{array}{c}
q^{1+3 k} c, q^{1+3 k} e \\
q^{-1-3 k} a^{3} / c^{2} e^{2}
\end{array} \mid q^{3}\right]_{n} \frac{\left(q^{-2-3 k} a / c e ; q^{-1}\right)_{n}}{\left(q^{5+6 k} c e ; q^{6}\right)_{n}} \\
= & \frac{\left(a^{2} / c e ; q^{2}\right)_{2 n}}{\left[q c e / a, q^{3} c e / a ; q\right]_{n}}\left[\begin{array}{c}
q c^{2} e^{2} / a^{2} \\
a / q c, a / q e
\end{array} \mid q^{2}\right]_{n}\left[\begin{array}{c}
q c, q e \\
a^{3} / q c^{2} e^{2}
\end{array} \mid q^{3}\right]_{n} \frac{\left(a / q^{2} c e ; q^{-1}\right)_{n}}{\left(q^{5} c e ; q^{6}\right)_{n}} \\
& \times \frac{1-q^{3+3 n+9 k} c^{3} e^{3} / a^{3}}{1-q^{3+9 k} c^{3} e^{3} / a^{3}} \frac{\left(q^{1+2 n} c^{2} e^{2} / a^{2} ; q^{2}\right)_{3 k}\left(q^{5} c e ; q^{6}\right)_{k}}{\left(q c^{2} e^{2} / a^{2} ; q^{2}\right)_{3 k}\left(q^{5+6 n} c e ; q^{6}\right)_{k}} \\
& \times\left[\begin{array}{c}
q^{1+3 n} c, q^{1+3 n} e, q c e / a, q^{3} c e / a, q^{5} c e / a, q^{4-3 n} c^{2} e^{2} / a^{3} \\
q c, q e, q^{1+n} c e / a, q^{3+n} c e / a, q^{5+n} c e / a, q^{4} c^{2} e^{2} / a^{3}
\end{array} q^{3}\right]_{k}
\end{aligned}
$$

and then defining the finite quadratic sum in base $q^{3}$ by

$$
\begin{aligned}
V_{m}^{\diamond}(a, c, e)= & \sum_{k=0}^{m-1}\left(1-q^{3+9 k} c^{3} e^{3} / a^{3}\right)\left[\begin{array}{c}
q c, q e, q^{4} c^{2} e^{2} / a^{3} \\
q c e / a, q^{3} c e / a, q^{5} c e / a
\end{array} \mid q^{3}\right]_{k} \\
& \times\left[\begin{array}{c}
q c^{2} e^{2} / a^{2}, q^{3} c^{2} e^{2} / a^{2}, q^{5} c^{2} e^{2} / a^{2} \\
q^{5} c e, q^{8} c^{2} e^{3} / a^{3}, q^{8} c^{3} e^{2} / a^{3}
\end{array}\right]_{k} q^{3 k},
\end{aligned}
$$

we obtain the following transformation formula.

\section{Theorem 11 (Transformation between quartic and quadratic series).}

$$
\begin{aligned}
V_{n}(a, c, e)- & V_{n}\left(q^{3 m} a, q^{3 m} c, q^{3 m} e\right) \frac{\left[q c, q e, q c^{2} e^{2} / a^{3} ; q^{3}\right]_{m}\left(q c^{2} e^{2} / a^{2} ; q^{2}\right)_{3 m}}{\left[q^{5} c e, q^{2} c^{2} e^{3} / a^{3}, q^{2} c^{3} e^{2} / a^{3} ; q^{6}\right]_{m}(q c e / a ; q)_{3 m}} \\
= & \frac{(1-a / q c)(1-a / q e)\left(1-q c^{2} e^{2} / a^{3}\right)}{\left(1-a / q^{2} c e\right)\left(1-q^{2} c^{3} e^{2} / a^{3}\right)\left(1-q^{2} c^{2} e^{3} / a^{3}\right)}\left\{V_{m}^{\diamond}(a, c, e)-V_{m}^{\diamond}\left(q^{5 n} a, q^{3 n} c, q^{3 n} e\right)\right. \\
& \left.\times \frac{\left(a^{2} / c e ; q^{2}\right)_{2 n}}{\left[q c e / a, q^{3} c e / a ; q\right]_{n}}\left[\begin{array}{c}
q c^{2} e^{2} / a^{2} \\
a / q c, a / q e
\end{array} \mid q^{2}\right]_{n}\left[\begin{array}{c}
q c, q e \\
a^{3} / q c^{2} e^{2}
\end{array} \mid q^{3}\right]_{n} \frac{\left(a / q^{2} c e ; q^{-1}\right)_{n}}{\left(q^{5} c e ; q^{6}\right)_{n}}\right\} .
\end{aligned}
$$

Letting $n \rightarrow 1+m, c \rightarrow a / q$ and $e \rightarrow q^{-1-3 m}$ in this theorem, we derive the summation formula, which does not seem to have explicitly appeared previously.

\section{Corollary 12 (Terminating series identity).}

$$
\begin{gathered}
\sum_{k=0}^{m} \frac{1-q^{5 k} a}{1-a}\left[\begin{array}{c}
q^{-3-6 m} \\
q^{2}, q^{2+3 m} a
\end{array} \mid q^{2}\right]_{k}\left[\begin{array}{c}
a, q^{-3 m} \\
q^{6+6 m} a
\end{array} \mid q^{3}\right]_{k} \frac{\left(q^{2+3 m} a ; q^{2}\right)_{2 k}}{\left(q^{-1-3 m} ; q\right)_{k}} \frac{(-1)^{k} q^{(2+3 m) k-\left(\begin{array}{c}
k \\
2
\end{array}\right)}}{\left(q^{3-3 m} a ; q^{6}\right)_{k}} \\
=\left[\begin{array}{c}
q^{6+3 m} a, q^{-3 m} / a \\
q^{2},
\end{array} q^{4} \mid q^{3}\right]_{m}\left[\begin{array}{c}
q^{5}, \\
q^{9+3 m} a, q^{3-3 m} / a
\end{array} \mid q^{6}\right]_{m} .
\end{gathered}
$$




\subsection{Quartic series to cubic series}

Define two sequences by

$$
\begin{aligned}
\mathrm{A}_{k} & =\frac{\left(a^{2} / q c^{2} e ; q\right)_{k}\left(q^{3} c^{2} e^{2} / a^{2} ; q^{2}\right)_{k}\left(q^{4} c ; q^{3}\right)_{k}\left(q^{4} a^{2} / c e ; q^{4}\right)_{k}}{\left(q^{6} c^{2} e / a ; q^{4}\right)_{k}\left(q^{2} a^{3} / c^{2} e^{2} ; q^{3}\right)_{k}\left(q a / c ; q^{2}\right)_{k}(q c e / a ; q)_{k}}, \\
\mathrm{~B}_{k} & =\frac{\left[q^{2} a^{2} / c e, q^{6} c^{2} e / a ; q^{4}\right]_{k}\left(q e ; q^{3}\right)_{k}\left(a / q c e ; q^{-1}\right)_{k}}{\left[q^{2} c e / a, a^{2} / q^{2} c^{2} e ; q\right]_{k}\left(q^{3} a / e ; q^{2}\right)_{k}\left(q^{5} c e ; q^{6}\right)_{k}}
\end{aligned}
$$

It is not hard to check the relations

$$
\begin{aligned}
\varpi:= & \mathrm{A}_{-1} \mathrm{~B}_{0}=\frac{(1-c e / a)(1-a / q c)\left(1-a^{3} / q c^{2} e^{2}\right)\left(1-q^{2} c^{2} e / a\right)}{\left(1-a^{2} / q^{2} c^{2} e\right)\left(1-q c^{2} e^{2} / a^{2}\right)(1-q c)\left(1-a^{2} / c e\right)}, \\
\mathcal{R}:= & \frac{\mathrm{A}_{n-1} \mathrm{~B}_{n}}{\mathrm{~A}_{-1} \mathrm{~B}_{0}}=\frac{1-q^{2+4 n} c^{2} e / a}{1-q^{2} c^{2} e / a}\left[\begin{array}{c}
q c^{2} e^{2} / a^{2} \\
a / q c, q^{3} a / e
\end{array} \mid q^{2}\right]_{n} \\
& \times \frac{\left(a^{2} / c e ; q^{2}\right)_{2 n}}{\left[c e / a, q^{2} c e / a ; q\right]_{n}}\left[\begin{array}{c}
q c, q e \\
a^{3} / q c^{2} e^{2} \mid q^{3}
\end{array}\right]_{n} \frac{\left(a / q c e ; q^{-1}\right)_{n}}{\left(q^{5} c e ; q^{6}\right)_{n}}
\end{aligned}
$$

and compute the finite differences

$$
\begin{aligned}
& \nabla \mathrm{A}_{k}=\frac{\left(1-q^{5 k} a\right)\left(1-q^{1+k} c e / a\right)\left(1-q^{1+2 k} a / e\right)\left(1-q^{2} c^{3} e^{2} / a^{3}\right)}{\left(1-q^{2} c^{2} e / a^{2}\right)\left(1-q c^{2} e^{2} / a^{2}\right)(1-q c)\left(1-a^{2} / c e\right)} \\
& \times\left[\begin{array}{c}
a^{2} / q^{2} c^{2} e \\
q c e / a
\end{array} \mid q\right]_{k}\left[\begin{array}{c}
q c^{2} e^{2} / a^{2} \\
q a / c
\end{array} \mid q^{2}\right]_{k}\left[\begin{array}{c}
q c \\
q^{2} a^{3} / c^{2} e^{2} \mid q^{3}
\end{array}\right]_{k}\left[\begin{array}{c}
a^{2} / c e \\
q^{6} c^{2} e / a
\end{array} \mid q^{4}\right]_{k} q^{k}, \\
& \Delta \mathrm{B}_{k}=-\frac{\left(1-q^{4+5 k} a\right)\left(1-q^{1-2 k} c / a\right)\left(1-q^{3+2 k} c^{2} e^{2} / a^{2}\right)\left(1-q^{4+3 k} c\right)}{\left(1-q^{2} c e / a\right)\left(1-q^{2} c^{2} e / a^{2}\right)\left(1-q^{3} a / e\right)\left(1-q^{5} c e\right)} \\
& \times \frac{\left[q^{2} a^{2} / c e, q^{6} c^{2} e / a ; q^{4}\right]_{k}\left(q e ; q^{3}\right)_{k}\left(a / q c e ; q^{-1}\right)_{k}}{\left[q^{3} c e / a, a^{2} / q c^{2} e ; q\right]_{k}\left(q^{5} a / e ; q^{2}\right)_{k}\left(q^{11} c e ; q^{6}\right)_{k}} q^{k} .
\end{aligned}
$$

Then by means of the modified Abel lemma on summation by parts, the $V$-sum can be reformulated as follows:

$$
\begin{aligned}
& V_{n}(a, c, e) \frac{(1-q c e / a)(1-q a / e)\left(1-q^{2} c^{3} e^{2} / a^{3}\right)}{\left(1-q^{2} c^{2} e / a^{2}\right)\left(1-q c^{2} e^{2} / a^{2}\right)(1-q c)\left(1-a^{2} / c e\right)} \\
&=\sum_{k=0}^{n-1} \mathrm{~B}_{k} \nabla \mathrm{A}_{k}=\varpi\{\mathcal{R}-1\}-\sum_{k=0}^{n-1} \mathrm{~A}_{k} \Delta \mathrm{B}_{k} .
\end{aligned}
$$

By expressing the last partial sum in terms of $V$-sum as

$$
-\sum_{k=0}^{n-1} \mathrm{~A}_{k} \Delta \mathrm{B}_{k}=\frac{V_{n}\left(q^{4} a, q^{6} c, e\right)(1-q c / a)\left(1-q^{3} c^{2} e^{2} / a^{2}\right)\left(1-q^{4} c\right)}{\left(1-q^{2} c e / a\right)\left(1-q^{2} c^{2} e / a^{2}\right)\left(1-q^{3} a / e\right)\left(1-q^{5} c e\right)},
$$

we derive after some simplification the following relation

$$
\begin{aligned}
V_{n}(a, c, e)= & V_{n}\left(q^{4} a, q^{6} c, e\right) \frac{\left(q c ; q^{3}\right)_{2}\left(q c^{2} e^{2} / a^{2} ; q^{2}\right)_{2}(1-q c / a)\left(1-a^{2} / c e\right)}{(q c e / a ; q)_{2}\left(q a / e ; q^{2}\right)_{2}\left(1-q^{5} c e\right)\left(1-q^{2} c^{3} e^{2} / a^{3}\right)} \\
& +\{1-\mathcal{R}(a, c, e)\} \frac{(1-c e / a)(1-q c / a)\left(1-a^{3} / q c^{2} e^{2}\right)\left(1-q^{2} c^{2} e / a\right)}{(1-a / q c e)(1-q a / e)\left(1-q^{2} c^{3} e^{2} / a^{3}\right)}
\end{aligned}
$$

Iterating it $m$-times, we get the following expression

$$
V_{n}(a, c, e)=V_{n}\left(q^{4 m} a, q^{6 m} c, e\right) \frac{\left(q c ; q^{3}\right)_{2 m}\left(q c^{2} e^{2} / a^{2} ; q^{2}\right)_{2 m}\left[q c / a, a^{2} / c e ; q^{2}\right]_{m}}{(q c e / a ; q)_{2 m}\left(q a / e ; q^{2}\right)_{2 m}\left[q^{5} c e, q^{2} c^{3} e^{2} / a^{3} ; q^{6}\right]_{m}}
$$




$$
\begin{aligned}
& +\frac{(1-c e / a)(1-q c / a)\left(1-a^{3} / q c^{2} e^{2}\right)}{(1-a / q c e)(1-q a / e)\left(1-q^{2} c^{3} e^{2} / a^{3}\right)} \sum_{k=0}^{m-1} \frac{\left(1-q^{2+8 k} c^{2} e / a\right)\left(q c ; q^{3}\right)_{2 k}}{\left[q^{5} c e, q^{8} c^{3} e^{2} / a^{3} ; q^{6}\right]_{k}} \\
& \times\left\{1-\mathcal{R}\left(q^{4 k} a, q^{6 k} c, e\right)\right\}\left[\begin{array}{l}
q^{3} c / a, a^{2} / c e \\
c e / a, q^{3} c e / a
\end{array} \mid q^{2}\right]_{k} \frac{\left(q c^{2} e^{2} / a^{2} ; q^{2}\right)_{2 k}}{\left(q^{3} a / e ; q^{2}\right)_{2 k}} q^{2 k} .
\end{aligned}
$$

Rewriting the $\mathcal{R}$-function explicitly as

$$
\begin{aligned}
\mathcal{R}\left(q^{4 k} a, q^{6 k} c, e\right)= & \frac{1-q^{2+4 n+8 k} c^{2} e / a}{1-q^{2+8 k} c^{2} e / a}\left[\begin{array}{c}
q^{1+4 k} c^{2} e^{2} / a^{2} \\
q^{-1-2 k} a / c, q^{3+4 k} a / e
\end{array} \mid q^{2}\right]_{n} \\
& \times \frac{\left(q^{2 k} a^{2} / c e ; q^{2}\right)_{2 n}}{\left[q^{2 k} c e / a, q^{2+2 k} c e / a ; q\right]_{n}}\left[\begin{array}{c}
q^{1+6 k} c, q e \\
a^{3} / q c^{2} e^{2}
\end{array} \mid q^{3}\right]_{n} \frac{\left(q^{-1-2 k} a / c e ; q^{-1}\right)_{n}}{\left(q^{5+6 k} c e ; q^{6}\right)_{n}} \\
= & \frac{\left(a^{2} / c e ; q^{2}\right)_{2 n}}{\left[c e / a, q^{2} c e / a ; q\right]_{n}}\left[\begin{array}{c}
q c^{2} e^{2} / a^{2} \\
a / q c, q^{3} a / e
\end{array} \mid q^{2}\right]_{n}\left[\begin{array}{c}
q c, q e \\
a^{3} / q c^{2} e^{2}
\end{array} \mid q^{3}\right]_{n} \frac{\left(a / q c e ; q^{-1}\right)_{n}}{\left(q^{5} c e ; q^{6}\right)_{n}} \\
& \times \frac{1-q^{2+4 n+8 k} c^{2} e / a}{1-q^{2+8 k} c^{2} e / a}\left[\begin{array}{c}
c e / a, q^{3} c e / a, q^{4 n} a^{2} / c e, q^{3-2 n} c / a \\
q^{n} c e / a, q^{n+3} c e / a, a^{2} / c e, q^{3} c / a
\end{array} \mid q^{2}\right]_{k} \\
& \times \frac{\left(q^{1+3 n} c ; q^{3}\right)_{2 k}\left(q^{5} c e ; q^{6}\right)_{k}}{\left(q c ; q^{3}\right)_{2 k}\left(q^{5+6 n} c e ; q^{6}\right)_{k}}\left[\begin{array}{c}
q^{1+2 n} c^{2} e^{2} / a^{2}, q^{3} a / e \\
q c^{2} e^{2} / a^{2}, q^{3+2 n} a / e
\end{array} \mid q^{2}\right]_{2 k},
\end{aligned}
$$

and defining further the finite cubic sum in base $q^{2}$ by

$$
V_{m}^{\triangle}(a, c, e)=\sum_{k=0}^{m-1}\left(1-q^{2+8 k} c^{2} e / a\right)\left[\begin{array}{l}
q^{3} c / a, a^{2} / c e \\
c e / a, q^{3} c e / a
\end{array} \mid q^{2}\right]_{k} \frac{\left(q c ; q^{3}\right)_{2 k}\left(q c^{2} e^{2} / a^{2} ; q^{2}\right)_{2 k}}{\left(q^{3} a / e ; q^{2}\right)_{2 k}\left[q^{5} c e, q^{8} c^{3} e^{2} / a^{3} ; q^{6}\right]_{k}} q^{2 k}
$$

we establish the following transformation formula.

\section{Theorem 13 (Transformation between quartic and cubic series).}

$$
\begin{aligned}
V_{n}(a, c, e)- & V_{n}\left(q^{4 m} a, q^{6 m} c, e\right) \frac{\left(q c ; q^{3}\right)_{2 m}\left(q c^{2} e^{2} / a^{2} ; q^{2}\right)_{2 m}\left[q c / a, a^{2} / c e ; q^{2}\right]_{m}}{(q c e / a ; q)_{2 m}\left(q a / e ; q^{2}\right)_{2 m}\left[q^{5} c e, q^{2} c^{3} e^{2} / a^{3} ; q^{6}\right]_{m}} \\
= & \frac{(1-c e / a)(1-q c / a)\left(1-a^{3} / q c^{2} e^{2}\right)}{(1-a / q c e)(1-q a / e)\left(1-q^{2} c^{3} e^{2} / a^{3}\right)}\left\{V_{m}^{\triangle}(a, c, e)-V_{m}^{\triangle}\left(q^{5 n} a, q^{3 n} c, q^{3 n} e\right)\right. \\
& \left.\times \frac{\left(a^{2} / c e ; q^{2}\right)_{2 n}}{\left[c e / a, q^{2} c e / a ; q\right]_{n}}\left[\begin{array}{c}
q c^{2} e^{2} / a^{2} \\
a / q c, q^{3} a / e
\end{array} \mid q^{2}\right]_{n}\left[\begin{array}{c}
q c, q e \\
a^{3} / q c^{2} e^{2}
\end{array} \mid q^{3}\right]_{n} \frac{\left(a / q c e ; q^{-1}\right)_{n}}{\left(q^{5} c e ; q^{6}\right)_{n}}\right\} .
\end{aligned}
$$

When $n \rightarrow 1+\delta+2 m, e \rightarrow q^{-1 / 2} a^{3 / 2} / c$ and $c=q^{-1-3 \delta-6 m}$ with $\delta=0,1$, the last theorem recovers the following summation formula.

\section{Corollary 14 (Chu [4, Equation (4.8d)]).}

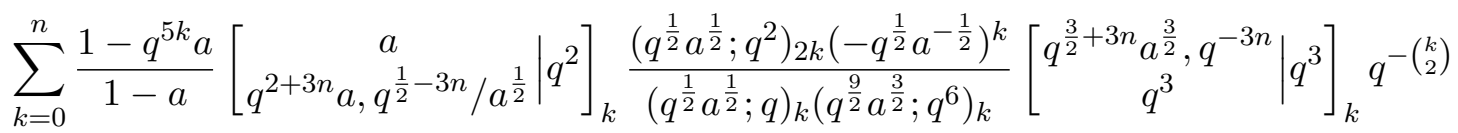

$$
\begin{aligned}
& = \begin{cases}0, & n \text {-odd; } \\
{\left[\begin{array}{c}
q^{2} a \\
q^{3 / 2} a^{3 / 2} \mid q^{2}
\end{array}\right]_{3 m}\left[\begin{array}{c}
q^{3} \\
q^{9 / 2} a^{3 / 2} \mid q^{6}
\end{array}\right]_{m}, \quad n=2 m .}\end{cases}
\end{aligned}
$$

Instead, letting $n \rightarrow 1+m, e \rightarrow q^{-1 / 2} a^{3 / 2} / c$ and $a=q^{-1-4 m}$ in Theorem 13 , we recover another terminating series identity. 


\section{Corollary 15 (Chu and Wang [8, Corollary 40]).}

$$
\begin{gathered}
\sum_{k=0}^{m} \frac{1-q^{5 k-1-4 m}}{1-q^{-1-4 m}}\left[\begin{array}{c}
q^{-1-4 m} \\
q^{-4 m} / c, q^{2+2 m} c \mid
\end{array} \mid q^{2}\right]_{k} \frac{\left(q^{-2 m} ; q^{2}\right)_{2 k}\left(-q^{1+2 m}\right)^{k}}{\left(q^{-2 m} ; q\right)_{k}\left(q^{3-6 m} ; q^{6}\right)_{k}}\left[\begin{array}{c}
q c, q^{-1-6 m} \\
q^{3}
\end{array} \mid q^{3}\right]_{k} q^{-\left(\begin{array}{c}
k \\
2
\end{array}\right)} \\
=\left[\begin{array}{c}
q^{4} c \\
q^{3}
\end{array} \mid q^{6}\right]_{m}\left[\begin{array}{c}
q \\
q^{2} c
\end{array} \mid q^{2}\right]_{2 m}\left[\begin{array}{c}
q^{2} c \\
q
\end{array} \mid q^{2}\right]_{m} .
\end{gathered}
$$

\subsection{Quartic series to quartic series}

Finally, for the two sequences given by

$$
\begin{aligned}
\mathrm{A}_{k} & =\frac{\left[q^{4} a^{2} / c e, q^{2} c e^{2} / a ; q^{4}\right]_{k}\left(q^{4} c ; q^{3}\right)_{k}\left(a / c e ; q^{-1}\right)_{k}}{\left[q c e / a, q^{3} a^{2} / c e^{2} ; q\right]_{k}\left(q a / c ; q^{2}\right)_{k}\left(q^{5} c e ; q^{6}\right)_{k}} \\
\mathrm{~B}_{k} & =\frac{\left(q^{3} a^{2} / c e^{2} ; q\right)_{k}\left(c^{2} e^{2} / q a^{2} ; q^{2}\right)_{k}\left(e / q^{2} ; q^{3}\right)_{k}\left(q^{2} a^{2} / c e ; q^{4}\right)_{k}}{\left(c e^{2} / q^{2} a ; q^{4}\right)_{k}\left(q^{2} a^{3} / c^{2} e^{2} ; q^{3}\right)_{k}\left(q^{3} a / e ; q^{2}\right)_{k}(c e / q a ; q)_{k}} ;
\end{aligned}
$$

we have no difficulty to check the relations

$$
\begin{aligned}
\varpi:= & \mathrm{A}_{-1} \mathrm{~B}_{0}=\frac{(1-c e / a)\left(1-q^{2} a^{2} / c e^{2}\right)(1-a / q c)(1-c e / q)}{\left(1-a^{2} / c e\right)\left(1-c e^{2} / q^{2} a\right)(1-q c)(1-q a / c e)}, \\
\mathcal{R}:= & \frac{\mathrm{A}_{n-1} \mathrm{~B}_{n}}{\mathrm{~A}_{-1} \mathrm{~B}_{0}}=\frac{1-q^{2+n} a^{2} / c e^{2}}{1-q^{2} a^{2} / c e^{2}}\left[\begin{array}{c}
c^{2} e^{2} / q a^{2} \\
a / q c, q^{3} a / e
\end{array} \mid q^{2}\right]_{n} \\
& \times \frac{\left(a^{2} / c e ; q^{2}\right)_{2 n}}{[c e / q a, c e / a ; q]_{n}}\left[\begin{array}{c}
q c, e / q^{2} \\
q^{2} a^{3} / c^{2} e^{2}
\end{array} \mid q^{3}\right]_{n} \frac{\left(q a / c e ; q^{-1}\right)_{n}}{\left(c e / q ; q^{6}\right)_{n}}
\end{aligned}
$$

and compute the finite differences

$$
\begin{aligned}
\nabla \mathrm{A}_{k}= & \frac{\left(1-q^{5 k} a\right)\left(1-q^{3 k-2} e\right)\left(1-q^{1+2 k} a / e\right)\left(1-q^{2 k-1} c^{2} e^{2} / a^{2}\right)}{\left(1-a^{2} / c e\right)\left(1-c e^{2} / q^{2} a\right)(1-q c)(1-c e / q a)} \\
& \times \frac{\left[a^{2} / c e, c e^{2} / q^{2} a ; q^{4}\right]_{k}\left(q c ; q^{3}\right)_{k}\left(q a / c e ; q^{-1}\right)_{k}}{\left[q c e / a, q^{3} a^{2} / c e^{2} ; q\right]_{k}\left(q a / c ; q^{2}\right)_{k}\left(q^{5} c e ; q^{6}\right)_{k}} \\
\Delta \mathrm{B}_{k}= & -\frac{\left(1-q^{1+5 k} a\right)\left(1-q^{k} c e / a\right)\left(1-q^{2 k-1} a / c\right)\left(1-q^{4} a^{3} / c^{2} e^{3}\right)}{(1-q a / c e)\left(1-q^{3} a / e\right)\left(1-q^{2} a^{3} / c^{2} e^{2}\right)\left(1-c e^{2} / q^{2} a\right)} \\
& \times\left[\begin{array}{c}
q^{3} a^{2} / c e^{2} \\
c e / a
\end{array} \mid q\right]_{k}\left[\begin{array}{c}
c^{2} e^{2} / q a^{2} \\
q^{5} a / e
\end{array} \mid q^{2}\right]_{k}\left[\begin{array}{c}
e / q^{2} \\
q^{5} a^{3} / c^{2} e^{2} \mid q^{3}
\end{array}\right]_{k}\left[\begin{array}{c}
q^{2} a^{2} / c e \\
q^{2} c e^{2} / a
\end{array} \mid q^{4}\right]_{k} q^{k} .
\end{aligned}
$$

Then according to the modified Abel lemma on summation by parts, the $V$-sum can be reformulated as follows:

$$
\begin{array}{r}
V_{n}(a, c, e) \frac{\left(1-e / q^{2}\right)(1-q a / e)\left(1-c^{2} e^{2} / q a^{2}\right)}{\left(1-a^{2} / c e\right)\left(1-c e^{2} / q^{2} a\right)(1-q c)(1-c e / q a)} \\
\quad=\sum_{k=0}^{n-1} \mathrm{~B}_{k} \nabla \mathrm{A}_{k}=\varpi\{\mathcal{R}-1\}-\sum_{k=0}^{n-1} \mathrm{~A}_{k} \Delta \mathrm{B}_{k} .
\end{array}
$$

Observing that the last partial sum results in

$$
-\sum_{k=0}^{n-1} \mathrm{~A}_{k} \Delta \mathrm{B}_{k}=\frac{V_{n}\left(q a, q^{3} c, e / q^{3}\right)(1-c e / a)(1-a / q c)\left(1-q^{4} a^{3} / c^{2} e^{3}\right)}{(1-q a / c e)\left(1-q^{3} a / e\right)\left(1-q^{2} a^{3} / c^{2} e^{2}\right)\left(1-c e^{2} / q^{2} a\right)},
$$

we derive after some simplification the following relation

$$
V_{n}(a, c, e)=a \frac{(1-a / c e)\left(1-q^{2} a^{2} / c e^{2}\right)(1-q c / a)(1-q / c e)}{\left(1-q a^{2} / c^{2} e^{2}\right)\left(1-q^{2} / e\right)(1-q a / e)}\{\mathcal{R}(a, c, e)-1\}
$$




$$
-V_{n}\left(q a, q^{3} c, e / q^{3}\right) \frac{(1-q c)(1-q c / a)(1-a / c e)\left(1-a^{2} / c e\right)\left(1-q^{4} a^{3} / c^{2} e^{3}\right)}{(c e / q a)\left(1-q^{2} a^{3} / c^{2} e^{2}\right)\left(1-q a^{2} / c^{2} e^{2}\right)\left(1-q^{2} / e\right)\left(q a / e ; q^{2}\right)_{2}} .
$$

Iterating it $m$-times, we get the following expression

$$
\begin{aligned}
V_{n}(a, c, e)= & V_{n}\left(q^{m} a, q^{3 m} c, q^{-3 m} e\right)\left[\begin{array}{c}
q c / a, a^{2} / c e \\
q a^{2} / c^{2} e^{2}
\end{array} \mid q^{2}\right]_{m} \\
& \times \frac{[a / c e, q a / c e ; q]_{m}}{\left(q a / e ; q^{2}\right)_{2 m}}\left[\begin{array}{c}
q c \\
q^{2} a^{3} / c^{2} e^{2}, q^{2} / e
\end{array} \mid q^{3}\right]_{n} \frac{\left(q^{4} a^{3} / c^{2} e^{3} ; q^{6}\right)_{m}}{\left(c e / q a ; q^{-1}\right)_{m}} \\
& -a \frac{(1-a / c e)(1-q c / a)(1-q / c e)}{\left(1-q a^{2} / c^{2} e^{2}\right)\left(1-q^{2} / e\right)(1-q a / e)} \\
& \times \sum_{k=0}^{m-1}\left(1-q^{2+5 k} a^{2} / c e^{2}\right)\left[\begin{array}{c}
q^{3} c / a, a^{2} / c e \\
q^{3} a^{2} / c^{2} e^{2}
\end{array} \mid q^{2}\right]_{k} \frac{(q a / c e ; q)_{k}}{\left(q^{3} a / e ; q^{2}\right)_{2 k}} q^{\left(\begin{array}{c}
k \\
2
\end{array}\right)} \\
& \times\left\{1-\mathcal{R}\left(q^{k} a, q^{3 k} c, q^{-3 k} e\right)\right\} \frac{\left(q c ; q^{3}\right)_{k}\left(q^{4} a^{3} / c^{2} e^{3} ; q^{6}\right)_{k}}{\left[q^{2} a^{3} / c^{2} e^{2}, q^{5} / e ; q^{3}\right]_{k}}\left(-\frac{q^{2} a}{c e}\right)^{k} .
\end{aligned}
$$

Writing explicitly the $\mathcal{R}$-function as

$$
\begin{aligned}
\mathcal{R}\left(q^{k} a, q^{3 k} c, q^{-3 k} e\right)= & \frac{1-q^{2+n+5 k} a^{2} / c e^{2}}{1-q^{2+5 k} a^{2} / c e^{2}}\left[\begin{array}{c}
q^{-1-2 k} c^{2} e^{2} / a^{2} \\
q^{-1-2 k} a / c, q^{3+4 k} a / e
\end{array} \mid q^{2}\right]_{n} \\
& \times \frac{\left(q^{2 k} a^{2} / c e ; q^{2}\right)_{2 n}}{\left[q^{-1-k} c e / a, q^{-k} c e / a ; q\right]_{n}}\left[\begin{array}{c}
q^{1+3 k} c, q^{-2-3 k} e \\
q^{2+3 k} a^{3} / c^{2} e^{2}
\end{array} \mid q^{3}\right]_{n} \frac{\left(q^{1+k} a / c e ; q^{-1}\right)_{n}}{\left(c e / q ; q^{6}\right)_{n}} \\
= & \frac{\left(a^{2} / c e ; q^{2}\right)_{2 n}}{[c e / q a, c e / a ; q]_{n}}\left[\begin{array}{c}
c^{2} e^{2} / q a^{2} \\
a / q c, q^{3} a / e
\end{array} \mid q^{2}\right]_{n}\left[\begin{array}{c}
q c, e / q^{2} \\
q^{2} a^{3} / c^{2} e^{2}
\end{array} \mid q^{3}\right]_{n} \frac{\left(q a / c e ; q^{-1}\right)_{n}}{\left(c e / q ; q^{6}\right)_{n}} \\
& \times \frac{1-q^{2+n+5 k} a^{2} / c e^{2}}{1-q^{5 k+2} a^{2} / c e^{2}}\left[\begin{array}{c}
q^{3} a^{2} / c^{2} e^{2}, q^{4 n} a^{2} / c e, q^{3-2 n} c / a \\
q^{3-2 n} a^{2} / c^{2} e^{2}, a^{2} / c e, q^{3} c / a
\end{array} \mid q^{2}\right]_{k} q^{-n k} \\
& \times \frac{\left(q^{1-n} a / c e ; q\right)_{k}\left(q^{3} a / e ; q^{2}\right)_{2 k}}{(q a / c e ; q)_{k}\left(q^{3+2 n} a / e ; q^{2}\right)_{2 k}}\left[\begin{array}{c}
q^{1+3 n} c, q^{5} / e, q^{2} a^{3} / c^{2} e^{2} \\
\left.q c, q^{5-3 n} / e, q^{2+3 n} a^{3} / c^{2} e^{2} \mid q^{3}\right]_{k}
\end{array}\right.
\end{aligned}
$$

and then defining the finite sum of quartic series

$$
\begin{aligned}
V_{m}^{\star}(a, c, e)= & \sum_{k=0}^{m-1}\left(1-q^{2+5 k} a^{2} / c e^{2}\right)\left[\begin{array}{c}
q^{3} c / a, a^{2} / c e \\
q^{3} a^{2} / c^{2} e^{2}
\end{array} \mid q^{2}\right]_{k} \frac{(q a / c e ; q)_{k}}{\left(q^{3} a / e ; q^{2}\right)_{2 k}} q^{\left(\begin{array}{c}
k \\
2
\end{array}\right)} \\
& \times\left(-\frac{q^{2} a}{c e}\right)^{k}\left[\begin{array}{c}
q c \\
q^{2} a^{3} / c^{2} e^{2}, q^{5} / e
\end{array} \mid q^{3}\right]_{k}\left(q^{4} a^{3} / c^{2} e^{3} ; q^{6}\right)_{k}
\end{aligned}
$$

we find the following transformation formula.

\section{Theorem 16 (Transformation between two quartic series).}

$$
\begin{aligned}
& V_{n}(a, c, e)-V_{n}\left(q^{m} a, q^{3 m} c, q^{-3 m} e\right)\left[\begin{array}{c}
q c / a, a^{2} / c e \\
q a^{2} / c^{2} e^{2} \mid q^{2}
\end{array}\right]_{m} \\
& \times \frac{[a / c e, q a / c e ; q]_{m}}{\left(q a / e ; q^{2}\right)_{2 m}}\left[\begin{array}{c}
q c \\
q^{2} a^{3} / c^{2} e^{2}, q^{2} / e
\end{array} \mid q^{3}\right]_{n} \frac{\left(q^{4} a^{3} / c^{2} e^{3} ; q^{6}\right)_{m}}{\left(c e / q a ; q^{-1}\right)_{m}} \\
& =\frac{(1-a / q c)(1-a / c e)(1-c e / q)}{\left(1-q a^{2} / c^{2} e^{2}\right)\left(1-e / q^{2}\right)(1-q a / e)}\left\{V_{m}^{\star}(a, c, e)-V_{m}^{\star}\left(q^{5 n} a, q^{3 n} c, q^{3 n} e\right)\right. \\
& \left.\times \frac{\left(a^{2} / c e ; q^{2}\right)_{2 n}}{[c e / q a, c e / a ; q]_{n}}\left[\begin{array}{c}
c^{2} e^{2} / q a^{2} \\
a / q c, q^{3} a / e
\end{array} \mid q^{2}\right]_{n}\left[\begin{array}{c}
q c, e / q^{2} \\
q^{2} a^{3} / c^{2} e^{2} \mid q^{3}
\end{array}\right]_{n} \frac{\left(q a / c e ; q^{-1}\right)_{n}}{\left(c e / q ; q^{6}\right)_{n}}\right\} .
\end{aligned}
$$


Letting $n \rightarrow 1+m, c=q^{-1-3 m}$ and $e \rightarrow q^{2+3 m}$, we obtain from the last theorem the following terminating series identity.

\section{Corollary 17 (Terminating series identity).}

$$
\begin{aligned}
& \sum_{k=0}^{m} \frac{1-q^{5 k} a}{1-a}\left[\begin{array}{c}
q^{3} / a^{2} \\
q^{2+3 m} a, q^{-1-3 m} a
\end{array} \mid q^{2}\right]_{k}\left[\begin{array}{c}
q^{3+3 m}, q^{-3 m} \\
a^{3}
\end{array} \mid q^{3}\right]_{k} \frac{\left(a^{2} / q ; q^{2}\right)_{2 k}(-a)^{k}}{\left(q^{2} / a ; q\right)_{k}\left(q^{6} ; q^{6}\right)_{k}} q^{-\left(\begin{array}{c}
1+k \\
2
\end{array}\right)} \\
& =\frac{[a / q, q a ; q]_{m}\left(q^{-3 m} / a ; q^{2}\right)_{m}\left(q^{-3 m} a^{3} ; q^{6}\right)_{m}}{\left(q^{-1-3 m} a ; q^{2}\right)_{2 m}\left(a^{3} ; q^{3}\right)_{m}\left(1 / a ; q^{-1}\right)_{m}}
\end{aligned}
$$

\section{Concluding remarks}

Recently, hypergeometric series has been found to have elliptic analogue after the pioneering work of Frenkel-Turaev [9]. Warnaar [20] derived several terminating elliptic series identities. Further summation formulae have been established by Chu-Jia [6] through Abel's lemma on summation by parts. It is plausible that the same approach works also for the elliptic analogue of the quartic series. All what we have gotten are two terminating elliptic analogues for Corollary 6 plus one for Corollary 17. Following the notations of [6], they are produced below for reader's reference.

Theorem 18 (Terminating elliptic series identity).

$$
\begin{aligned}
& \sum_{k=0}^{m} \frac{\theta\left(q^{5 k} a ; p\right)}{\theta(a ; p)}\left[\begin{array}{c}
a, q^{2+m} \\
q^{1-2 m}
\end{array} \mid q^{2}, p\right]_{k} \frac{\left[q^{-m} ; q, p\right]_{k}\left[q^{1-m} a ; q^{6}, p\right]_{k}}{\left[q^{2+m} a ; q^{2}, p\right]_{2 k}} q^{\left(\begin{array}{c}
1+k \\
2
\end{array}\right)-m k} \\
& \times(-1)^{k}\left[\begin{array}{c}
q^{1+2 m} a \\
q^{3}, q^{1-m} a
\end{array} \mid q^{3}, p\right]_{k}=\frac{\chi(m=2 n)\left[q^{2} a ; q^{2}, p\right]_{n}\left[q^{3} ; q^{6}, p\right]_{n}}{\left[q^{1+2 n} ; q^{2}, p\right]_{n}\left[q^{4-2 n} a ; q^{6}, p\right]_{n}} .
\end{aligned}
$$

Theorem 19 (Terminating elliptic series identity).

$$
\begin{aligned}
& \sum_{k=0}^{m} \frac{\theta\left(q^{5 k} a ; p\right)}{\theta(a ; p)}\left[\begin{array}{c}
a, q^{-2 m} \\
q^{5+4 m}
\end{array} \mid q^{2}, p\right]_{k} \frac{\left[q^{2+2 m} ; q, p\right]_{k}\left[q^{3+2 m} a ; q^{6}, p\right]_{k}}{\left[q^{-2 m} a ; q^{2}, p\right]_{2 k}} q^{\left(\begin{array}{c}
k \\
2
\end{array}\right)+(3+2 m) k} \\
& \quad \times(-1)^{k}\left[\begin{array}{c}
q^{-3-4 m} a \\
q^{3}, q^{3+2 m} a
\end{array} \mid q^{3}, p\right]_{k}=\frac{\left[q^{2} a ; q^{2}, p\right]_{m}\left[q^{5} ; q^{2}, p\right]_{2 m}\left[q^{-4 m} a ; q^{6}, p\right]_{m}}{\left[q^{3} ; q^{2}, p\right]_{m}\left[q^{-2 m} a ; q^{2}, p\right]_{2 m}\left[q^{9} ; q^{6}, p\right]_{m}} .
\end{aligned}
$$

Theorem 20 (Terminating elliptic series identity).

$$
\begin{aligned}
& \sum_{k=0}^{m} \frac{\theta\left(q^{5 k} a ; p\right)}{\theta(a ; p)}\left[\begin{array}{c}
q^{3} / a^{2} \\
q^{2+3 m} a, q^{-1-3 m} a
\end{array} \mid q^{2}, p\right]_{k} \frac{\left[a^{2} / q ; q^{2}, p\right]_{2 k}(-a)^{k}}{\left[q^{2} / a ; q, p\right]_{k}\left[q^{6} ; q^{6}, p\right]_{k}} q^{-\left(\begin{array}{c}
1+k \\
2
\end{array}\right)} \\
& \quad \times\left[\begin{array}{c}
q^{3+3 m}, q^{-3 m} \\
a^{3}
\end{array} \mid q^{3}, p\right]_{k}=\frac{[a / q, q a ; q, p]_{m}\left[q^{-3 m} / a ; q^{2}, p\right]_{m}\left[q^{-3 m} a^{3} ; q^{6}, p\right]_{m}}{\left[q^{-1-3 m} a ; q^{2}, p\right]_{2 m}\left[a^{3} ; q^{3}, p\right]_{m}\left[1 / a ; q^{-1}, p\right]_{m}} .
\end{aligned}
$$

\section{Acknowledgments}

The work of the second author was partially supported by National Science Foundation of China (Youth grant 10801026). 


\section{References}

[1] Andrews G.E., Three aspects of partitions, Séminaire Lotharingien de Combinatoire (Salzburg, 1990), Publ. Inst. Rech. Math. Av., Vol. 462, Univ. Louis Pasteur, Strasbourg, 1991, 5-18.

[2] Andrews G.E., Askey R., Roy R., Special functions, Encyclopedia of Mathematics and Its Applications, Vol. 71, Cambridge University Press, Cambridge, 1999.

[3] Bailey W.N., Well-poised basic hypergeometric series, Quart. J. Math. Oxford Ser. 18 (1947), 157-166.

[4] Chu W., Inversion techniques and combinatorial identities: Jackson's $q$-analogue of the Dougall-Dixon theorem and the dual formulae, Compositio Math. 95 (1995), 43-68.

[5] Chu W., Abel's lemma on summation by parts and basic hypergeometric series, Adv. in Appl. Math. 39 (2007), 490-514.

[6] Chu W., Jia C., Abel's method on summation by parts and theta hypergeometric series, J. Combin. Theory Ser. A 115 (2008), 815-844.

[7] Chu W., Wang C., Abel's lemma on summation by parts and partial $q$-series transformations, Sci. China Ser. A 52 (2009), 720-748.

[8] Chu W., Wang X., Abel's lemma on summation by parts and terminating $q$-series identities, Numer. Algorithms 49 (2008), 105-128.

[9] Frenkel I.B., Turaev V.G., Elliptic solutions of the Yang-Baxter equation and modular hypergeometric functions, The Arnold-Gelfand Mathematical Seminars, Birkhäuser Boston, Boston, MA, 1997, 171-204.

[10] Gasper G., Summation, transformation, and expansion formulas for bibasic series, Trans. Amer. Math. Soc. 312 (1989), 257-277.

[11] Gasper G., Rahman M., An indefinite bibasic summation formula and some quadratic, cubic and quartic summation and transformation formulas, Canad. J. Math. 42 (1990), 1-27.

[12] Gasper G., Rahman M., Basic hypergeometric series, 2nd ed., Encyclopedia of Mathematics and Its Applications, Vol. 96, Cambridge University Press, Cambridge, 2004.

[13] Gessel I., Stanton D., Applications of $q$-Lagrange inversion to basic hypergeometric series, Trans. Amer. Math. Soc. 277 (1983), 173-201.

[14] Ismail M.E.H., Stanton D., Tribasic integrals and identities of Rogers-Ramanujan type, Trans. Amer. Math. Soc. 355 (2003), 4061-4091.

[15] Koornwinder T.H., Askey-Wilson polynomials as zonal spherical functions on the SU(2) quantum group, SIAM J. Math. Anal. 24 (1993), 795-813.

[16] Rahman M., Some quadratic and cubic summation formulas for basic hypergeometric series, Canad. J. Math. 45 (1993), 394-411.

[17] Rahman M., Verma A., Quadratic transformation formulas for basic hypergeometric series, Trans. Amer. Math. Soc. 335 (1993), 277-302.

[18] Stanton D., The Bailey-Rogers-Ramanujan group, Contemp. Math. 291 (2001), 55-70.

[19] Stromberg K.R., An introduction to classical real analysis, Wadsworth International Mathematics Series, Wadsworth International, Belmont, Calif., 1981.

[20] Warnaar S.O., Summation and transformation formulas for elliptic hypergeometric series, Constr. Approx. 18 (2002), 479-502, math.QA/0001006. 